\title{
Pressure from the vacuum of confined spinor matter
}

\author{
Yu. A. Sitenko ${ }^{1,2}$ and S. A. Yushchenko ${ }^{1}$ \\ ${ }^{1}$ Bogolyubov Institute for Theoretical Physics, \\ National Academy of Sciences of Ukraine, \\ 14-b Metrologichna Str., 03680 Kyiv, Ukraine \\ ${ }^{2}$ Institute for Theoretical Physics, University of Bern, \\ Sidlerstrasse 5, CH-3012 Bern, Switzerland
}

\begin{abstract}
Charged spinor matter field is quantized in a spatial region bounded by two parallel neutral plates. The most general set of boundary conditions ensuring the confinement of matter within the plates is considered. We study a response of the vacuum of the confined matter to the background uniform magnetic field which is directed orthogonally to the plates. It is proven that, in the case of a sufficiently strong magnetic field, the vacuum pressure onto the plates is positive and independent of the boundary condition, as well as of the distance between the plates.
\end{abstract}

PACS: 03.70.+k, 11.10.-z, 12.20.Ds

Keywords: boundary conditions, confined matter, background magnetic field, Casimir effect 


\section{Introduction}

Perhaps, a quest for boundary conditions ensuring the confinement of the quantized spinor matter was initiated in the context of a model description of hadrons as composite systems with their internal structure being associated with quark-gluon constituents [1, 2, 3, 4]. If an hadron is an extended object occupying spatial region $\Omega$ bounded by surface $\partial \Omega$, then the condition that the quark matter field be confined inside the hadron is formulated as

$$
\left.\boldsymbol{n} \cdot \boldsymbol{J}(\mathbf{r})\right|_{\mathbf{r} \in \partial \Omega}=0
$$

where $\boldsymbol{n}$ is the unit normal to the boundary surface, and $\boldsymbol{J}(\mathbf{r})=\psi^{\dagger}(\mathbf{r}) \boldsymbol{\alpha} \psi(\mathbf{r})$ with $\psi(\mathbf{r})(\mathbf{r} \in \Omega)$ being the quark matter field $\left(\alpha^{1}, \alpha^{2}, \alpha^{3}\right.$ and $\beta$ are the generating elements of the Dirac-Clifford algebra); an appropriate condition is also formulated for the gluon matter field.

The concept of confined matter fields is quite familiar in the context of condensed matter physics: collective excitations (e.g., spin waves and phonons) exist only inside material objects and do not spread outside. Moreover, in the context of quantum electrodynamics, if one is interested in the effect of a classical background magnetic field on the vacuum of the quantized electron-positron matter, then the latter should be considered as confined to the spatial region between the sources of the magnetic field, as long as collective quasielectronic excitations inside a magnetized material differ from electronic excitations in the vacuum. It should be noted in this respect that the study of the effect of the background electromagnetic field on the vacuum of quantized charged matter has begun already eight decades ago [5, 6, 7, 8, 9], see review in [10]. However, the concern has been for the case of a background field filling the whole (infinite) space, that is hard to be regarded as realistic. The case of both the background and quantized fields confined to a bounded spatial region with boundaries serving as sources of the background field looks much more physically plausible, it can even be regarded as realizable in laboratory. Moreover, there is no way to detect the energy density that is induced in the vacuum in the first case, whereas the pressure from the vacuum onto the boundaries, resulting in the second case, is in principle detectable.

In view of the above, an issue of a choice of boundary conditions for the quantized matter fields gains a crucial significance, and condition (1) should be resolved to take the form of a boundary condition that is linear in $\psi(\mathbf{r})$. 
Recall that an immediate way of such a resolution is known as the MIT bag boundary condition [4],

$$
\left.[I+\mathrm{i} \beta(\boldsymbol{n} \cdot \boldsymbol{\alpha})] \psi(\mathbf{r})\right|_{\mathbf{r} \in \partial \Omega}=0,
$$

but it is needless to say that this way is not a unique one. The most general boundary condition that is linear in $\psi(\mathbf{r})$ in the case of a simply-connected boundary involves four arbitrary parameters [11, and the explicit form of this boundary condition has been given [12]. The condition is compatible with the self-adjointness of the differential operator of one-particle energy in first-quantized theory (Dirac hamiltonian operator in the case of relativistic spinor matter). The self-adjointness of operators of physical observables is required by general principles of comprehensibility and mathematical consistency, see, e.g., [13, 14]. To put it simply, a multiple action is well defined for a self-adjoint operator only, allowing for the construction of functions of the operator, such as resolvent, evolution, heat kernel and zeta-function operators, with further implications upon second quantization. In the present paper, we follow the lines of works [11, 12] by proposing a different, embracing more cases, form of the four-parameter generalization of the MIT bag boundary condition.

Thus, we consider in general the quantized spinor matter field that is confined to the three-dimensional spatial region $\Omega$ bounded by the twodimensional surface $\partial \Omega$. To study a response of the vacuum to the background magnetic field, we restrict ourselves to the case of the boundary consisting of two parallel planes; the magnetic field is assumed to be uniform and orthogonal to the planes. Such a spatial geometry is typical for the remarkable macroscopic quantum phenomenon which yields the attraction (negative pressure) between two neutral plates and which is known as the Casimir effect [15], see reviews in [16, 17]. The conventional Casimir effect is due to vacuum fluctuations of the quantized electromagnetic field obeying certain boundary conditions at the bounding plates, and a choice of boundary conditions is physically motivated by material properties of the plates (for instance, metallic or dielectric ones, see, e.g., [17]). Such a motivation is lacking for the case of vacuum fluctuations of the quantized spinor matter field. That is why there is a necessity in the last case to start from the most general set of mathematically acceptable (i.e. compatible with the self-adjointness) boundary conditions. Then follows, as has been already discussed, a physical constraint that spinor matter be confined within the plates. A further physical constraint, as will be shown in Section 4, is that the spectrum of 
the wave number vector in the direction which is orthogonal to the plates be real and unambiguously (although implicitly) determined. Employing these mathematical and physical restrictions, we explore the generalized Casimir effect which is due to vacuum fluctuations of the quantized spinor matter field in the presence of the background magnetic field; the pressure from the vacuum onto the bounding plates will be found.

In the next section we show how the requirement of the self-adjointness for the Dirac hamiltonian operator brings the most general set of boundary conditions ensuring the confinement of the quantized spinor matter in the cases of a simply-connected boundary and a disconnected boundary consisting of two noncompact noncontiguous surfaces. In Section 3 we consider the vacuum energy which is induced by a background uniform magnetic field in the cases of the unbounded quantization volume and the quantization volume bounded by two parallel infinite plates. The boundary condition determining unambiguously the spectrum of the wave number vector in the direction orthogonal to the plates is derived, and the general expression for the pressure from the vacuum onto the plates is obtained in Section 4. The vacuum pressure in some particular cases is examined in Section 5, while the asymptotical behaviour of the vacuum pressure at small and large separations of the plates is analysed in Section 6. Finally, the results are summarized and discussed in Section 7. We adduce the solution to the Dirac equation in the background uniform magnetic field in Appendix A. In Appendix B we derive a new version of the Abel-Plana formula for summation over values of the wave number vector in the direction orthogonal to the plates.

\section{Self-adjointness and boundary conditions}

Defining a scalar product as $(\tilde{\chi}, \chi)=\int_{\Omega} \mathrm{d}^{3} r \tilde{\chi}^{\dagger} \chi$, we get, using integration by parts,

$$
(\tilde{\chi}, H \chi)=\left(H^{\dagger} \tilde{\chi}, \chi\right)-\mathrm{i} \int_{\partial \Omega} \mathrm{d} \mathbf{s} \cdot \tilde{\chi}^{\dagger} \boldsymbol{\alpha} \chi,
$$

where

$$
H=H^{\dagger}=-\mathrm{i} \boldsymbol{\alpha} \cdot \boldsymbol{\nabla}+\beta m
$$

is the formal expression for the Dirac hamiltonian operator and $\boldsymbol{\nabla}$ is the covariant derivative involving both the affine and bundle connections (natural 
units $\hbar=c=1$ are used). Operator $H$ is Hermitian (or symmetric in mathematical parlance),

$$
(\tilde{\chi}, H \chi)=\left(H^{\dagger} \tilde{\chi}, \chi\right)
$$

if

$$
\int_{\partial \Omega} \mathrm{d} \mathbf{s} \cdot \tilde{\chi}^{\dagger} \boldsymbol{\alpha} \chi=0 .
$$

The latter condition can be satisfied in various ways by imposing different boundary conditions for $\chi$ and $\tilde{\chi}$. However, among the whole variety, there may exist a possibility that a boundary condition for $\tilde{\chi}$ is the same as that for $\chi$; then the domain of definition of $H^{\dagger}$ (set of functions $\tilde{\chi}$ ) coincides with that of $H$ (set of functions $\chi$ ), and operator $H$ is self-adjoint. The action of a self-adjoint operator results in functions belonging to its domain of definition only, and, therefore, a multiple action and functions of such an operator can be consistently defined.

Condition (6) is certainly fulfilled when the integrand in (6) vanishes, i.e.

$$
\left.\tilde{\chi}^{\dagger}(\boldsymbol{n} \cdot \boldsymbol{\alpha}) \chi\right|_{\mathbf{r} \in \partial \Omega}=0
$$

To fulfill the latter condition, we impose the same boundary condition for $\chi$ and $\tilde{\chi}$ in the form

$$
\left.\chi\right|_{\mathbf{r} \in \partial \Omega}=\left.K \chi\right|_{\mathbf{r} \in \partial \Omega},\left.\quad \tilde{\chi}\right|_{\mathbf{r} \in \partial \Omega}=\left.K \tilde{\chi}\right|_{\mathbf{r} \in \partial \Omega}
$$

where $K$ is a matrix (element of the Dirac-Clifford algebra) which is determined by two conditions:

$$
K^{2}=I
$$

and

$$
K^{\dagger}(\boldsymbol{n} \cdot \boldsymbol{\alpha}) K=-\boldsymbol{n} \cdot \boldsymbol{\alpha}
$$

It should be noted that, in addition to (7), the following combination of $\chi$ and $\tilde{\chi}$ is also vanishing at the boundary:

$$
\left.\tilde{\chi}^{\dagger}(\boldsymbol{n} \cdot \boldsymbol{\alpha}) K \chi\right|_{\mathbf{r} \in \partial \Omega}=\left.\tilde{\chi}^{\dagger} K^{\dagger}(\boldsymbol{n} \cdot \boldsymbol{\alpha}) \chi\right|_{\mathbf{r} \in \partial \Omega}=0 .
$$

Using the standard representation for the Dirac matrices,

$$
\beta=\left(\begin{array}{cc}
I & 0 \\
0 & -I
\end{array}\right), \quad \boldsymbol{\alpha}=\left(\begin{array}{cc}
0 & \boldsymbol{\sigma} \\
\boldsymbol{\sigma} & 0
\end{array}\right)
$$


$\left(\sigma^{1}, \sigma^{2}\right.$ and $\sigma^{3}$ are the Pauli matrices), one can get

$$
K=\left(\begin{array}{cc}
0 & \varrho^{-1} \\
\varrho & 0
\end{array}\right)
$$

where condition

$$
(\boldsymbol{n} \cdot \boldsymbol{\sigma}) \varrho=-\varrho^{\dagger}(\boldsymbol{n} \cdot \boldsymbol{\sigma})
$$

defines $\varrho$ as a rank-2 matrix depending on four arbitrary parameters [11]. An explicit form for matrix $K$ is [12]

$$
K=\frac{\left(1+u^{2}-v^{2}-\boldsymbol{t}^{2}\right) \beta+\left(1-u^{2}+v^{2}+\boldsymbol{t}^{2}\right) I}{2 \mathrm{i}\left(u^{2}-v^{2}-\boldsymbol{t}^{2}\right)}\left(u \boldsymbol{n} \cdot \boldsymbol{\alpha}+v \beta \gamma^{5}-\mathrm{i} \boldsymbol{t} \cdot \boldsymbol{\alpha}\right),
$$

where $\gamma^{5}=\mathrm{i} \alpha^{1} \alpha^{2} \alpha^{3}$, and $\boldsymbol{t}=\left(t^{1}, t^{2}\right)$ is a two-dimensional vector which is tangential to the boundary, $\boldsymbol{t} \cdot \boldsymbol{n}=0$. Matrix $K$ is Hermitian, $K^{\dagger}=K$, if either

$$
u=1, \quad v=0, \quad \boldsymbol{t}=0
$$

or

$$
u=0, \quad v^{2}+\boldsymbol{t}^{2}=1 .
$$

Using parametrization

$$
\begin{gathered}
u=\cosh \tilde{\vartheta} \cosh \vartheta, \quad v=\cosh \tilde{\vartheta} \sinh \vartheta \cos \theta \\
t^{1}=\cosh \tilde{\vartheta} \sinh \vartheta \sin \theta \cos \phi, \quad t^{2}=\cosh \tilde{\vartheta} \sinh \vartheta \sin \theta \sin \phi \\
-\infty<\vartheta<\infty, \quad 0 \leq \tilde{\vartheta}<\infty, \quad 0 \leq \theta<\pi, \quad 0 \leq \phi<2 \pi
\end{gathered}
$$

in the case of $u^{2}-v^{2}-\boldsymbol{t}^{2} \geq 1$, one gets

$$
\begin{gathered}
K=\frac{\beta\left(1+\cosh ^{2} \tilde{\vartheta}\right)-I \sinh ^{2} \tilde{\vartheta}}{2 \mathrm{i} \cosh \tilde{\vartheta}} \\
\left.\times\left[\boldsymbol{n} \cdot \boldsymbol{\alpha} \cosh \vartheta+\beta \gamma^{5} \sinh \vartheta \cos \theta-\mathrm{i}\left(\alpha^{1} \cos \phi+\alpha^{2} \sin \phi\right) \sinh \vartheta \sin \theta\right)\right],
\end{gathered}
$$

while, using parametrization

$$
\begin{gathered}
u=\cosh \tilde{\varsigma} \sinh \varsigma, \quad v=\cosh \tilde{\varsigma} \cosh \varsigma \cos \theta, \\
t^{1}=\cosh \tilde{\varsigma} \cosh \varsigma \sin \theta \cos \phi, \quad t^{2}=\cosh \tilde{\varsigma} \cosh \varsigma \sin \theta \sin \phi, \\
-\infty<\varsigma<\infty, \quad 0 \leq \tilde{\varsigma}<\infty, \quad 0 \leq \theta<\pi, \quad 0 \leq \phi<2 \pi
\end{gathered}
$$


in the case of $u^{2}-v^{2}-\boldsymbol{t}^{2} \leq-1$, one gets

$$
\begin{gathered}
K=\frac{\beta \sinh ^{2} \tilde{\varsigma}-I\left(1+\cosh ^{2} \tilde{\varsigma}\right)}{2 \mathrm{i} \cosh \tilde{\varsigma}} \\
\times\left[\boldsymbol{n} \cdot \boldsymbol{\alpha} \sinh \varsigma+\beta \gamma^{5} \cosh \varsigma \cos \theta-\mathrm{i}\left(\alpha^{1} \cos \phi+\alpha^{2} \sin \phi\right) \cosh \varsigma \sin \theta\right] ;
\end{gathered}
$$

here

$$
\left[\boldsymbol{n} \cdot \boldsymbol{\alpha}, \alpha^{1}\right]_{+}=\left[\boldsymbol{n} \cdot \boldsymbol{\alpha}, \alpha^{2}\right]_{+}=\left[\alpha^{1}, \alpha^{2}\right]_{+}=0 .
$$

The intermediate case of $-1 \leq u^{2}-v^{2}-\boldsymbol{t}^{2} \leq 1$ is obtained by going over to imaginary values of parameters $\tilde{\vartheta}$ and $\tilde{\varsigma}$ :

$$
\operatorname{Re} \tilde{\vartheta}=0, \quad 0 \leq \operatorname{Im} \tilde{\vartheta}<\pi / 2 \quad\left(0<u^{2}-v^{2}-\boldsymbol{t}^{2} \leq 1\right)
$$

and

$$
\operatorname{Re} \tilde{\varsigma}=0, \quad 0 \leq \operatorname{Im} \tilde{\varsigma}<\pi / 2 \quad\left(-1 \leq u^{2}-v^{2}-\boldsymbol{t}^{2}<0\right) .
$$

Parameters $\vartheta, \tilde{\vartheta}$ (or $\varsigma, \tilde{\varsigma}), \theta$ and $\phi$ can be interpreted as the self-adjoint extension parameters. It should be emphasized that the values of these parameters vary in general from point to point of the boundary. In this respect the "number" of self-adjoint extension parameters is in fact infinite, moreover, it is not countable but is of power of a continuum. This distinguishes the case of an extended boundary from the case of an excluded point (contact interaction), when the number of self-adjoint extension parameters is finite, being equal to $n^{2}$ for the deficiency index equal to $\{n, n\}$ (see, e.g., [14]).

At the points where matrix $K$ is Hermitian, it takes forms

$$
K_{+}=-\mathrm{i} \beta(\boldsymbol{n} \cdot \boldsymbol{\alpha}) \quad\left(u^{2}-v^{2}-\boldsymbol{t}^{2}=1\right)
$$

and

$$
K_{-}=\mathrm{i} \beta \gamma^{5} \cos \theta+\left(\alpha^{1} \cos \phi+\alpha^{2} \sin \phi\right) \sin \theta \quad\left(u^{2}-v^{2}-\boldsymbol{t}^{2}=-1\right) .
$$

A transition from $K_{+}$to $K_{-}$in the parametric space is performed with the use of (18) by varying $\tilde{\vartheta}$ from 0 to $\mathrm{i} \pi / 2$ and then with the use of (20) by varying $\tilde{\varsigma}$ from $i \pi / 2$ to 0 . Matrix $K_{+}(24)$ corresponds to the choice of the standard MIT bag boundary condition [4], cf (2),

$$
\left.\left(I-K_{+}\right) \chi\right|_{\mathbf{r} \in \partial \Omega}=\left.\left(I-K_{+}\right) \tilde{\chi}\right|_{\mathbf{r} \in \partial \Omega}=0,
$$

when relation (11) takes form

$$
\left.\tilde{\chi}^{\dagger} \beta \chi\right|_{\mathbf{r} \in \partial \Omega}=0 .
$$


To elucidate the meaning of the choice that is corresponded with matrix $K_{-}$ (25), one has to perform a transition from $K_{+}$to $K_{-}$in a parametric space with the same set of parameters, i.e. two different pairs of parameters, $(\vartheta, \tilde{\vartheta})$ and $(\varsigma, \tilde{\varsigma})$, should be changed to a single one, say, $(\varphi, \tilde{\varphi})$. The most natural way is to revoke the condition that $K$ be off-diagonal, imposing instead the condition that $K$ be Hermitian (as $K_{+}$and $K_{-}$are). Then, in view of relation (9), $K$ is unitary as well, $K^{\dagger}=K^{-1}$, and relation (10) is rewritten as

$$
[K, \boldsymbol{n} \cdot \boldsymbol{\alpha}]_{+}=0 .
$$

One can simply go through 16 linearly independent elements of the DiracClifford algebra and find 8 of them, which anticommute with $\boldsymbol{n} \cdot \boldsymbol{\alpha}$. Thus we get

$$
\begin{gathered}
K=c_{1} \alpha^{1}+c_{2} \alpha^{2}+\mathrm{i} c_{3} \alpha^{1}(\boldsymbol{n} \cdot \boldsymbol{\alpha})+\mathrm{i} c_{4} \alpha^{2}(\boldsymbol{n} \cdot \boldsymbol{\alpha}) \\
+c_{5} \beta+\mathrm{i} c_{6} \beta \gamma^{5}+\mathrm{i} c_{7} \beta(\boldsymbol{n} \cdot \boldsymbol{\alpha})+c_{8} \beta(\boldsymbol{n} \cdot \boldsymbol{\alpha}) \gamma^{5}
\end{gathered}
$$

where coefficients $c_{j}(j=\overline{1,8})$ are real, since $K$ is Hermitian, and, as a consequence of $(9)$, obey condition

$$
\frac{c_{1}}{c_{3}}=\frac{c_{2}}{c_{4}}=\frac{c_{5}}{c_{7}}=-\frac{c_{6}}{c_{8}} .
$$

Defining parameters $\varphi$ and $\tilde{\varphi}$ by arranging terms in (29) into combinations $\exp \left(\mathrm{i} \varphi \gamma^{5}\right)$ and $\exp (\mathrm{i} \tilde{\varphi} \boldsymbol{n} \cdot \boldsymbol{\alpha})$, where $-\pi / 2<\varphi \leq \pi / 2$ and $-\pi / 2 \leq \tilde{\varphi}<\pi / 2$, we recast (29) into the form

$$
K=\left(\tilde{c}_{1} \alpha^{1}+\tilde{c}_{2} \alpha^{2}+\tilde{c}_{3} \beta \mathrm{e}^{\mathrm{i} \varphi \gamma^{5}}\right) \mathrm{e}^{\mathrm{i} \tilde{\varphi} \boldsymbol{n} \cdot \boldsymbol{\alpha}}
$$

where real coefficients $\tilde{c}_{j}(j=\overline{1,3})$, in view of $(9)$, obey condition

$$
\tilde{c}_{1}^{2}+\tilde{c}_{2}^{2}+\tilde{c}_{3}^{2}=1
$$

With the use of obvious parametrization

$$
\tilde{c}_{1}=\sin \theta \cos \phi, \quad \tilde{c}_{2}=\sin \theta \sin \phi, \quad \tilde{c}_{3}=\cos \theta
$$

we finally obtain matrix $K$ in the form

$$
K=\left[\beta \mathrm{e}^{\mathrm{i} \varphi \gamma^{5}} \cos \theta+\left(\alpha^{1} \cos \phi+\alpha^{2} \sin \phi\right) \sin \theta\right] \mathrm{e}^{\mathrm{i} \tilde{\varphi} \boldsymbol{n} \cdot \boldsymbol{\alpha}},
$$

interpolating continuously (and smoothly) between $K_{+}$and $K_{-}$:

$$
K_{+}=\left.K\right|_{\varphi=0, \tilde{\varphi}=-\pi / 2, \theta=0}, \quad K_{-}=\left.K\right|_{\varphi=\pi / 2, \tilde{\varphi}=0} .
$$


The explicit form of the boundary condition ensuring the self-adjointness of operator $H(4)$ in this case is

$$
\left.\left\{I-\left[\beta \mathrm{e}^{\mathrm{i} \varphi \gamma^{5}} \cos \theta+\left(\alpha^{1} \cos \phi+\alpha^{2} \sin \phi\right) \sin \theta\right] \mathrm{e}^{\mathrm{i} \tilde{\varphi} \boldsymbol{n} \cdot \boldsymbol{\alpha}}\right\} \chi\right|_{\mathbf{r} \in \partial \Omega}=0
$$

(the same condition is for $\tilde{\chi}$ ), and relation (11) takes form

$$
\left.\tilde{\chi}^{\dagger}\left[\beta \mathrm{e}^{\mathrm{i} \varphi \gamma^{5}} \cos \theta+\left(\alpha^{1} \cos \phi+\alpha^{2} \sin \phi\right) \sin \theta\right] \mathrm{e}^{\mathrm{i}(\tilde{\varphi}+\pi / 2) \boldsymbol{n} \cdot \boldsymbol{\alpha}} \chi\right|_{\mathbf{r} \in \partial \Omega}=0 .
$$

Four parameters of boundary condition $(34), \varphi, \tilde{\varphi}, \theta$ and $\phi$, which vary arbitrarily from point to point of the boundary, are interpreted as the self-adjoint extension parameters.

In the context of the Casimir effect, one usually considers spatial region $\Omega$ with a disconnected boundary consisting of two connected components, $\partial \Omega=\partial \Omega^{(+)} \bigcup \partial \Omega^{(-)}$. Choosing coordinates $\mathbf{r}=(x, y, z)$ in such a way that $x$ and $y$ are tangential to the boundary, while $z$ is normal to it, we identify the position of $\partial \Omega^{( \pm)}$with, say, $z= \pm a / 2$. In general, there are 8 selfadjoint extension parameters: $\varphi_{+}, \tilde{\varphi}_{+}, \theta_{+}$and $\phi_{+}$corresponding to $\partial \Omega^{(+)}$and $\varphi_{-}, \tilde{\varphi}_{-}, \theta_{-}$and $\phi_{-}$corresponding to $\partial \Omega^{(-)}$. However, if some symmetry is present, then the number of self-adjoint extension parameters is diminished. For instance, if the boundary consists of two parallel planes, then the cases differing by the values of $\phi_{+}$or $\phi_{-}$are physically indistinguishable, since they are related by a rotation around a normal to the boundary. To avoid this unphysical degeneracy, one has to fix

$$
\theta_{+}=\theta_{-}=0
$$

and there remains 4 self-adjoint extension parameters: $\varphi_{+}, \tilde{\varphi}_{+}, \varphi_{-}$and $\tilde{\varphi}_{-}$. Operator $H(4)$ acting on functions which are defined in the region bounded by two parallel planes is self-adjoint, if the following condition holds:

$$
\left.\left\{I-\beta \exp \left[\mathrm{i}\left(\varphi_{ \pm} \gamma^{5} \pm \tilde{\varphi}_{ \pm} \alpha^{z}\right)\right]\right\} \chi\right|_{z= \pm a / 2}=0
$$

(the same condition holds for $\tilde{\chi}$ ). The latter ensures the fulfilment of constraints

$$
\left.\tilde{\chi}^{\dagger} \alpha^{z} \chi\right|_{z= \pm a / 2}=0
$$

and

$$
\left.\tilde{\chi}^{\dagger} \beta \exp \left\{\mathrm{i}\left[\varphi_{ \pm} \gamma^{5} \pm\left(\tilde{\varphi}_{ \pm}+\pi / 2\right) \alpha^{z}\right]\right\} \chi\right|_{z= \pm a / 2}=0
$$


It should be noted that, if one chooses the $K$-matrix to be non-Hermitian and off-diagonal in the standard representation, see (14), then, employing parametrization (17), the self-adjointness is implemented in the context of the Casimir effect with the use of boundary condition

$$
\left.\left[I-\frac{\beta\left(\cosh ^{2} \tilde{\vartheta}_{ \pm}+1\right)-I \sinh ^{2} \tilde{\vartheta}_{ \pm}}{2 \mathrm{i} \cosh \tilde{\vartheta}_{ \pm}}\left( \pm \alpha^{z} \cosh \vartheta_{ \pm}+\beta \gamma^{5} \sinh \vartheta_{ \pm}\right)\right] \chi\right|_{z= \pm a / 2}=0
$$

(the same condition is for $\tilde{\chi}$ ), while the analogue of (39) takes form

$$
\left.\frac{1}{2} \tilde{\chi}^{\dagger}\left[I\left(\cosh ^{2} \tilde{\vartheta}_{ \pm}+1\right)+\beta \sinh ^{2} \tilde{\vartheta}_{ \pm}\right]\left(\beta \cosh \vartheta_{ \pm} \mp \alpha^{z} \gamma^{5} \sinh \vartheta_{ \pm}\right) \chi\right|_{z= \pm a / 2}=0
$$

\section{Induced vacuum energy in the magnetic field background}

The operator of a spinor field which is quantized in an ultrastatic background is presented in the form

$$
\hat{\Psi}(t, \mathbf{r})=\bigvee_{E_{\lambda}>0} \mathrm{e}^{-\mathrm{i} E_{\lambda} t} \psi_{\lambda}(\mathbf{r}) \hat{a}_{\lambda}+\bigvee_{E_{\lambda}<0} \mathrm{e}^{-\mathrm{i} E_{\lambda} t} \psi_{\lambda}(\mathbf{r}) \hat{b}_{\lambda}^{\dagger}
$$

where $\hat{a}_{\lambda}^{\dagger}$ and $\hat{a}_{\lambda}\left(\hat{b}_{\lambda}^{\dagger}\right.$ and $\left.\hat{b}_{\lambda}\right)$ are the spinor particle (antiparticle) creation and destruction operators, satisfying anticommutation relations $\left[\hat{a}_{\lambda}, \hat{a}_{\lambda^{\prime}}^{\dagger}\right]_{+}=$ $\left[\hat{b}_{\lambda}, \hat{b}_{\lambda^{\prime}}^{\dagger}\right]_{+}=\left\langle\lambda \mid \lambda^{\prime}\right\rangle$, wave functions $\psi_{\lambda}(\mathbf{r})$ form a complete set of solutions to the stationary Dirac equation

$$
H \psi_{\lambda}(\mathbf{r})=E_{\lambda} \psi_{\lambda}(\mathbf{r})
$$

$\lambda$ is the set of parameters (quantum numbers) specifying a one-particle state with energy $E_{\lambda}$; symbol $\mathcal{L}$ denotes summation over discrete and integration (with a certain measure) over continuous values of $\lambda$. Ground state $\mid \mathrm{vac}>$ is defined by condition $\hat{a}_{\lambda}\left|\mathrm{vac}>=\hat{b}_{\lambda}\right| \mathrm{vac}>=0$. The temporal component of the operator of the energy-momentum tensor is given by expression

$$
\hat{T}^{00}=\frac{\mathrm{i}}{4}\left[\hat{\Psi}^{\dagger}\left(\partial_{0} \hat{\Psi}\right)-\left(\partial_{0} \hat{\Psi}^{T}\right) \hat{\Psi}^{\dagger T}-\left(\partial_{0} \hat{\Psi}^{\dagger}\right) \hat{\Psi}+\hat{\Psi}^{T}\left(\partial_{0} \hat{\Psi}^{\dagger T}\right)\right],
$$


where superscript $T$ denotes a transposed spinor. Consequently, the formal expression for the vacuum expectation value of the energy density is

$$
\varepsilon=<\operatorname{vac}\left|\hat{T}^{00}\right| \operatorname{vac}>=-\frac{1}{2} \bigvee\left|E_{\lambda}\right| \psi_{\lambda}^{\dagger}(\mathbf{r}) \psi_{\lambda}(\mathbf{r})
$$

Let us consider the quantized charged massive spinor field in the background of a static uniform magnetic field; then $\boldsymbol{\nabla}=\boldsymbol{\partial}-\mathrm{i} e \mathbf{A}$ and the connection can be chosen as $\mathbf{A}=(-y B, 0,0)$, where $B$ is the value of the magnetic field strength which is directed along the $z$-axis in Cartesian coordinates $\mathbf{r}=(x, y, z), \mathbf{B}=(0,0, B)$. The one-particle energy spectrum is

$$
E_{n k}= \pm \omega_{n k}
$$

where

$$
\omega_{n k}=\sqrt{2 n|e B|+k^{2}+m^{2}},-\infty<k<\infty, n=0,1,2, \ldots,
$$

$k$ is the value of the wave number vector along the $z$-axis, and $n$ labels the Landau levels. Using the explicit form of the complete set of solutions to the Dirac equation, see Appendix A, one can get that expression (45) takes form

$$
\varepsilon^{\infty}=-\frac{|e B|}{2 \pi^{2}} \int_{-\infty}^{\infty} \mathrm{d} k \sum_{n=0}^{\infty} \iota_{n} \omega_{n k}
$$

where $\iota_{n}=1-\frac{1}{2} \delta_{n 0}$; the superscript on the left-hand side indicates that the magnetic field fills the whole (infinite) space. The integral and the sum in (48) are divergent and require regularization and renormalization. This problem has been solved long ago by Heisenberg and Euler [7] (see also [9]), and we just list here their result

$$
\varepsilon_{\text {ren }}^{\infty}=\frac{1}{8 \pi^{2}} \int_{0}^{\infty} \frac{\mathrm{d} \tau}{\tau} \mathrm{e}^{-\tau}\left[\frac{e B m^{2}}{\tau} \operatorname{coth}\left(\frac{e B \tau}{m^{2}}\right)-\frac{m^{4}}{\tau^{2}}-\frac{1}{3} e^{2} B^{2}\right] ;
$$

note that the renormalization procedure includes subtraction at $B=0$ and renormalization of the charge.

Let us turn now to the quantized charged massive spinor field in the background of a static uniform magnetic field in spatial region $\Omega$ bounded by two parallel planes $\partial \Omega^{(+)}$and $\partial \Omega^{(-)}$; the position of $\partial \Omega^{( \pm)}$is identified 
with $z= \pm a / 2$, and the magnetic field is orthogonal to the boundary. The solution to (43) in region $\Omega$ is chosen as a superposition of two plane waves propagating in opposite directions along the $z$-axis,

$$
\psi_{q n l}(\mathbf{r})=\psi_{q n k_{l}}(\mathbf{r})+\psi_{q n-k_{l}}(\mathbf{r})
$$

where the explicit form of $\psi_{q n k}(\mathbf{r})$ and $\psi_{q n-k}(\mathbf{r})$ is given in Appendix A, and all restrictions on the values of coefficients $C_{j}$ and $\tilde{C}_{j}(j=0,1,2)$ are withdrawn for a while. The values of wave number vector $k_{l}(l=0, \pm 1, \pm 2, \ldots)$ are determined from the boundary condition, see (37):

$$
\left.\left\{I-\beta \exp \left[\mathrm{i}\left(\varphi_{ \pm} \gamma^{5} \pm \tilde{\varphi}_{ \pm} \alpha^{z}\right)\right]\right\} \psi_{q n l}(\mathbf{r})\right|_{z= \pm a / 2}=0, \quad n \geq 1
$$

and

$$
\begin{gathered}
{\left[I+\frac{\beta}{2}\left( \pm \alpha^{z} \gamma^{5}-1\right) \mathrm{e}^{\mathrm{i}\left(\varphi_{ \pm}-\tilde{\varphi}_{ \pm}\right) \gamma^{5}} \Theta( \pm e B)\right.} \\
\left.-\frac{\beta}{2}\left( \pm \alpha^{z} \gamma^{5}+1\right) \mathrm{e}^{\mathrm{i}\left(\varphi_{ \pm}+\tilde{\varphi}_{ \pm}\right) \gamma^{5}} \Theta(\mp e B)\right]\left.\psi_{q 0 l}^{(0)}(\mathbf{r})\right|_{z= \pm a / 2}=0
\end{gathered}
$$

where the step function is introduced as $\Theta(u)=1$ at $u>0$ and $\Theta(u)=$ 0 at $u<0$. The latter conditions can be rewritten as conditions on the coefficients:

$$
\left\{\begin{array}{l}
M_{11}^{(n)} C_{1}+M_{12}^{(n)} C_{2}+M_{13}^{(n)} \tilde{C}_{1}+M_{14}^{(n)} \tilde{C}_{2}=0 \\
M_{21}^{(n)} C_{1}+M_{22}^{(n)} C_{2}+M_{23}^{(n)} \tilde{C}_{1}+M_{24}^{(n)} \tilde{C}_{2}=0 \\
M_{31}^{(n)} C_{1}+M_{32}^{(n)} C_{2}+M_{33}^{(n)} \tilde{C}_{1}+M_{34}^{(n)} \tilde{C}_{2}=0 \\
M_{41}^{(n)} C_{1}+M_{42}^{(n)} C_{2}+M_{43}^{(n)} \tilde{C}_{1}+M_{44}^{(n)} \tilde{C}_{2}=0
\end{array}\right\}, \quad n \geq 1
$$

and

$$
\left\{\begin{array}{l}
M_{11}^{(0)} C_{0}+M_{12}^{(0)} \tilde{C}_{0}=0 \\
M_{21}^{(0)} C_{0}+M_{22}^{(0)} \tilde{C}_{0}=0
\end{array}\right\}
$$


where

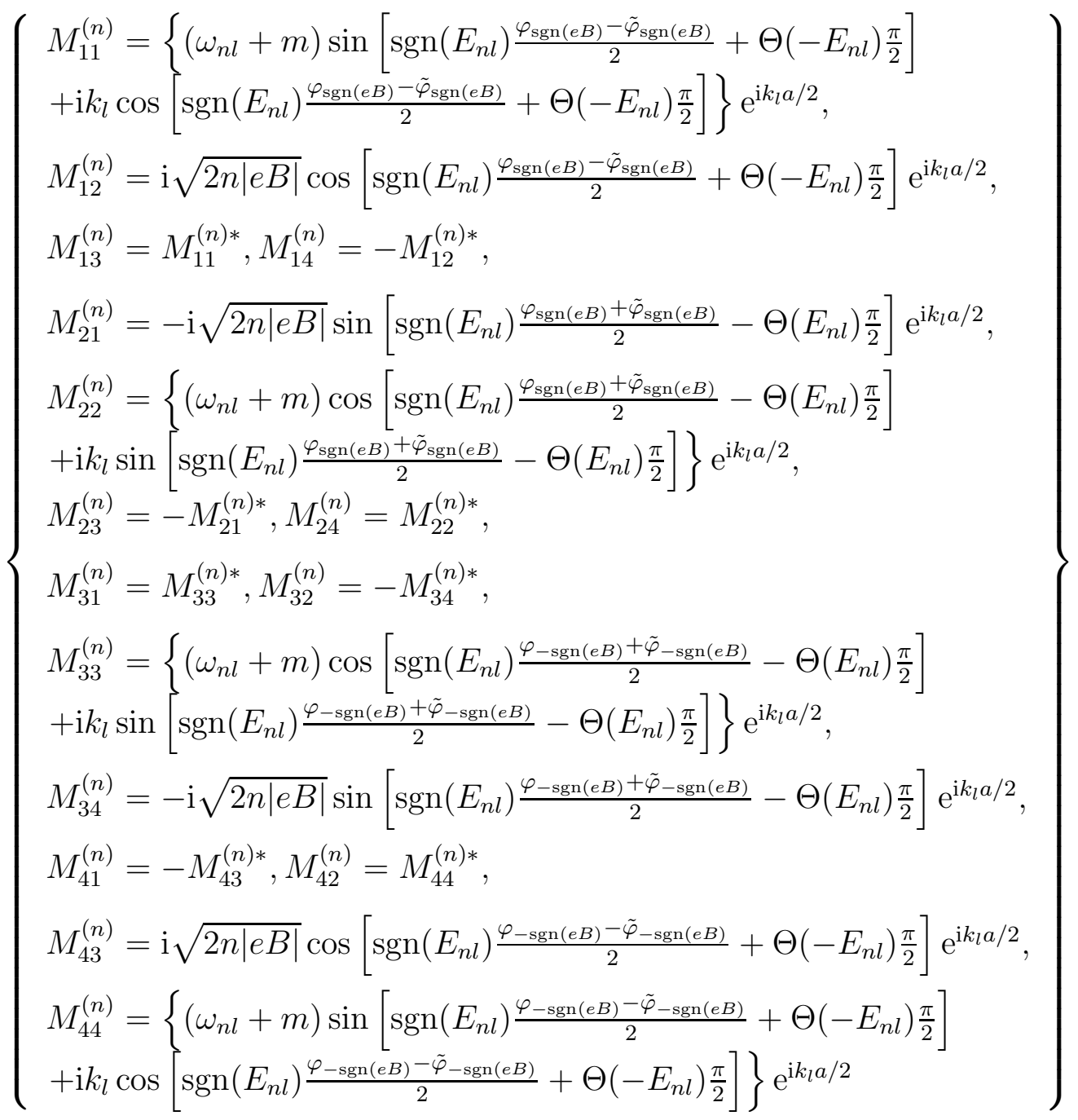

$$
\begin{aligned}
& n \geq 1
\end{aligned}
$$


and

$$
\left\{\begin{array}{l}
M_{11}^{(0)}=\left\{\left(\omega_{0 l}+m\right) \sin \left[\operatorname{sgn}\left(E_{0 l}\right) \frac{\varphi_{\operatorname{sgn}(e B)}-\tilde{\varphi}_{\operatorname{sgn}(e B)}}{2}+\Theta\left(-E_{0 l}\right) \frac{\pi}{2}\right]\right. \\
\left.+\mathrm{i} k_{l} \cos \left[\operatorname{sgn}\left(E_{0 l}\right) \frac{\varphi_{\operatorname{sgn}(e B)}-\tilde{\varphi}_{\operatorname{sgn}(e B)}}{2}+\Theta\left(-E_{0 l}\right) \frac{\pi}{2}\right]\right\} \mathrm{e}^{\mathrm{i} k_{l} a / 2}, \\
M_{12}^{(0)}=M_{11}^{(0) *}, M_{21}^{(0)}=M_{22}^{(0) *} \\
M_{22}^{(0)}=\left\{\left(\omega_{0 l}+m\right) \cos \left[\operatorname{sgn}\left(E_{0 l}\right) \frac{\varphi_{-\operatorname{sgn}(e B)}+\tilde{\varphi}_{-\operatorname{sgn}(e B)}}{2}-\Theta\left(E_{0 l}\right) \frac{\pi}{2}\right]\right. \\
\left.+\mathrm{i} k_{l} \sin \left[\operatorname{sgn}\left(E_{0 l}\right) \frac{\varphi_{-\operatorname{sgn}(e B)}+\tilde{\varphi}_{-\operatorname{sgn}(e B)}}{2}-\Theta\left(E_{0 l}\right) \frac{\pi}{2}\right]\right\} \mathrm{e}^{\mathrm{i} k_{l} a / 2}
\end{array}\right\} ;
$$

here, $\operatorname{sgn}(u)=\Theta(u)-\Theta(-u)$ is the sign function, we have chosen

$$
\alpha^{z}=\left(\begin{array}{cc}
0 & \sigma^{3} \\
\sigma^{3} & 0
\end{array}\right)
$$

and introduced notations

$$
\omega_{n l} \equiv \omega_{n k_{l}}=\sqrt{2 n|e B|+k_{l}^{2}+m^{2}}
$$

and, similarly, $E_{n l} \equiv E_{n k_{l}}$.

Thus, the spectrum of wave number vector $k_{l}$ is determined from condition

$$
\operatorname{det} M^{(n)}=0,
$$

where 


$$
\begin{gathered}
\operatorname{det} M^{(n)}=\left(m+\omega_{n l}\right)^{2} \\
\times\left\{\mathrm{e}^{2 \mathrm{i} k_{l} a}\left[m \cos \varphi_{+}-\omega_{n l} \operatorname{sgn}\left(E_{n l}\right) \cos \tilde{\varphi}_{+}-\mathrm{i} k_{l} \sin \tilde{\varphi}_{+}\right]\right. \\
\times\left[m \cos \varphi_{-}-\omega_{n l} \operatorname{sgn}\left(E_{n l}\right) \cos \tilde{\varphi}_{-}-\mathrm{i} k_{l} \sin \tilde{\varphi}_{-}\right] \\
-2\left[m \cos \varphi_{+}-\omega_{n l} \operatorname{sgn}\left(E_{n l}\right) \cos \tilde{\varphi}_{+}\right]\left[m \cos \varphi_{-}-\omega_{n l} \operatorname{sgn}\left(E_{n l}\right) \cos \tilde{\varphi}_{-}\right] \\
-2 k_{l}^{2}\left[\cos \left(\varphi_{+}-\varphi_{-}\right)-\cos \tilde{\varphi}_{+} \cos \tilde{\varphi}_{-}\right] \\
+\mathrm{e}^{-2 \mathrm{i} k_{l} a}\left[m \cos \varphi_{+}-\omega_{n l} \operatorname{sgn}\left(E_{n l}\right) \cos \tilde{\varphi}_{+}+\mathrm{i} k_{l} \sin \tilde{\varphi}_{+}\right] \\
\left.\times\left[m \cos \varphi_{-}-\omega_{n l} \operatorname{sgn}\left(E_{n l}\right) \cos \tilde{\varphi}_{-}+\mathrm{i} k_{l} \sin \tilde{\varphi}_{-}\right]\right\}, \quad n \geq 1
\end{gathered}
$$

and

$$
\begin{gathered}
\operatorname{det} M^{(0)}=\cos \left[\operatorname{sgn}\left(E_{0 l}\right) \frac{\varphi_{\operatorname{sgn}(e B)}-\tilde{\varphi}_{\operatorname{sgn}(e B)}}{2}+\Theta\left(-E_{0 l}\right) \frac{\pi}{2}\right] \\
\times \sin \left[\operatorname{sgn}\left(E_{0 l}\right) \frac{\varphi_{-\operatorname{sgn}(e B)}+\tilde{\varphi}_{-\operatorname{sgn}(e B)}}{2}-\Theta\left(E_{0 l}\right) \frac{\pi}{2}\right] \\
\times\left\{\mathrm{e}^{\mathrm{i} k_{l} a}\left[\left(m+\omega_{0 l}\right) \tan \left(\operatorname{sgn}\left(E_{0 l}\right) \frac{\varphi_{\operatorname{sgn}(e B)}-\tilde{\varphi}_{\operatorname{sgn}(e B)}}{2}+\Theta\left(-E_{0 l}\right) \frac{\pi}{2}\right)+\mathrm{i} k_{l}\right]\right. \\
\times\left[\left(m+\omega_{0 l}\right) \cot \left(\operatorname{sgn}\left(E_{0 l}\right) \frac{\varphi_{-\operatorname{sgn}(e B)}+\tilde{\varphi}_{-\operatorname{sgn}(e B)}}{2}-\Theta\left(E_{0 l}\right) \frac{\pi}{2}\right)+\mathrm{i} k_{l}\right] \\
-\mathrm{e}^{-\mathrm{i} k_{l} a}\left[\left(m+\omega_{0 l}\right) \tan \left(\operatorname{sgn}\left(E_{0 l}\right) \frac{\varphi_{\operatorname{sgn}(e B)}-\tilde{\varphi}_{\operatorname{sgn}(e B)}}{2}+\Theta\left(-E_{0 l}\right) \frac{\pi}{2}\right)-\mathrm{i} k_{l}\right] \\
\left.\times\left[\left(m+\omega_{0 l}\right) \cot \left(\operatorname{sgn}\left(E_{0 l}\right) \frac{\varphi_{-\operatorname{sgn}(e B)}+\tilde{\varphi}_{-\operatorname{sgn}(e B)}}{2}-\Theta\left(E_{0 l}\right) \frac{\pi}{2}\right)-\mathrm{i} k_{l}\right]\right\} .(60)
\end{gathered}
$$
$\tilde{C}_{0}$ :

Given solution $\psi_{q 0 l}^{(0)}(\mathbf{r})$, we impose the condition on its coefficients $C_{0}$ and

$$
\left\{\begin{array}{l}
\left|C_{0}\right|^{2}+\left|\tilde{C}_{0}\right|^{2}=\frac{2 \pi}{a}, \\
C_{0}^{*} \tilde{C}_{0}+\tilde{C}_{0}^{*} C_{0}=0 ;
\end{array}\right.
$$


in particular, the coefficients can be chosen as

$$
C_{0}=\sqrt{\frac{\pi}{a}} \mathrm{e}^{\mathrm{i} \pi / 4}, \quad \tilde{C}_{0}=\sqrt{\frac{\pi}{a}} \mathrm{e}^{-\mathrm{i} \pi / 4}
$$

In the case of $n \geq 1$, two linearly independent solutions, $\psi_{q n l}^{(1)}(\mathbf{r})$ and $\psi_{q n l}^{(2)}(\mathbf{r})$, are orthogonal, if the appropriate coefficients, $C_{j}^{(1)}, \tilde{C}_{j}^{(1)}$ and $C_{j}^{(2)}, \tilde{C}_{j}^{(2)}(j=$ $1,2)$, obey condition

$$
\left\{\begin{array}{l}
\sum_{j=1,2} C_{j}^{(1) *} C_{j}^{(2)}=0 \\
C_{j}^{(1)} C_{j^{\prime}}^{(2)}=\tilde{C}_{j}^{(1)} \tilde{C}_{j^{\prime}}^{(2)},\left|C_{j}^{\left(j^{\prime}\right)}\right|=\left|\tilde{C}_{j}^{\left(j^{\prime}\right)}\right|, \quad j, j^{\prime}=1,2
\end{array}\right.
$$

We impose further condition:

$$
\left\{\begin{array}{l}
\sum_{j=1,2}\left|C_{j}^{\left(j^{\prime}\right)}\right|^{2}=\frac{\pi}{a} \\
\sum_{j=1,2}\left[C_{j}^{\left(j^{\prime}\right) *} \tilde{C}_{j}^{\left(j^{\prime}\right)}+\tilde{C}_{j}^{\left(j^{\prime}\right) *} C_{j}^{\left(j^{\prime}\right)}\right]=0, \quad j^{\prime}=1,2
\end{array}\right.
$$

in particular, the coefficients can be chosen as

$$
C_{1}^{(1)}=\sqrt{\frac{\pi}{2 a}} \mathrm{e}^{\mathrm{i} \pi / 4}, \tilde{C}_{1}^{(1)}=\sqrt{\frac{\pi}{2 a}} \mathrm{e}^{-\mathrm{i} \pi / 4}, C_{2}^{(1)}=\sqrt{\frac{\pi}{2 a}} \mathrm{e}^{-\mathrm{i} \pi / 4}, \tilde{C}_{2}^{(1)}=\sqrt{\frac{\pi}{2 a}} \mathrm{e}^{-3 \mathrm{i} \pi / 4}
$$

and

$$
C_{1}^{(2)}=\sqrt{\frac{\pi}{2 a}} \mathrm{e}^{-\mathrm{i} \pi / 4}, \tilde{C}_{1}^{(2)}=\sqrt{\frac{\pi}{2 a}} \mathrm{e}^{\mathrm{i} \pi / 4}, C_{2}^{(2)}=\sqrt{\frac{\pi}{2 a}} \mathrm{e}^{\mathrm{i} \pi / 4}, \tilde{C}_{2}^{(2)}=\sqrt{\frac{\pi}{2 a}} \mathrm{e}^{3 \mathrm{i} \pi / 4} .
$$

As a result, wave functions $\psi_{q n l}^{(j)}(\mathbf{r})(j=0,1,2)$ satisfy the requirements of orthonormality

$$
\int_{\Omega} \mathrm{d}^{3} r \psi_{q n l}^{(j) \dagger}(\mathbf{r}) \psi_{q^{\prime} n^{\prime} l^{\prime}}^{\left(j^{\prime}\right)}(\mathbf{r})=\delta_{j j^{\prime}} \delta_{n n^{\prime}} \delta_{l l^{\prime}} \delta\left(q-q^{\prime}\right), \quad j, j^{\prime}=0,1,2
$$

and completeness

$$
\sum_{\operatorname{sgn}\left(E_{n l}\right)} \int_{-\infty}^{\infty} \mathrm{d} q \sum_{l}\left[\psi_{q 0 l}^{(0)}(\mathbf{r}) \psi_{q 0 l}^{(0) \dagger}\left(\mathbf{r}^{\prime}\right)+\sum_{n=1}^{\infty} \sum_{j=1,2} \psi_{q n l}^{(j)}(\mathbf{r}) \psi_{q n l}^{(j) \dagger}\left(\mathbf{r}^{\prime}\right)\right]=I \delta\left(\mathbf{r}-\mathbf{r}^{\prime}\right) .
$$


Consequently, we obtain the following formal expression for the vacuum expectation value of the energy per unit area of the boundary surface

$$
\frac{E}{S} \equiv \int_{-a / 2}^{a / 2} \mathrm{~d} z \varepsilon=-\frac{|e B|}{2 \pi} \sum_{\operatorname{sgn}\left(E_{n l}\right)} \sum_{l} \sum_{n=0}^{\infty} \iota_{n} \omega_{n l} .
$$

Concluding this section we recall that, owing to the boundary condition, see (51) and (52), the normal component of current $\boldsymbol{J}_{q n l j}(\mathbf{r})=\psi_{q n l}^{(j) \dagger}(\mathbf{r}) \boldsymbol{\alpha} \psi_{q n l}^{(j)}(\mathbf{r})$ $(j=0,1,2)$ vanishes at the boundary, see (38),

$$
\left.J_{q n l j}^{z}(\mathbf{r})\right|_{z= \pm a / 2}=0
$$

which, cf. (1), signifies that the quantized matter is confined within the boundaries.

\section{Choice of boundary conditions, Casimir energy and force}

The spectrum of the wave number vector in the direction of the magnetic field, which is determined from (58), depends on four self-adjoint extension parameters, $\varphi_{+}, \tilde{\varphi}_{+}, \varphi_{-}$and $\tilde{\varphi}_{-}$, in the case of $n \geq 1$, see (59), and on two self-adjoint extension parameters, $\varphi_{+}-\tilde{\varphi}_{+}$and $\varphi_{-}+\tilde{\varphi}_{-}(e B>0)$, or $\varphi_{+}+\tilde{\varphi}_{+}$ and $\varphi_{-}-\tilde{\varphi}_{-}(e B<0)$, in the case of $n=0$, see (60). As was mentioned

in Section 2, the values of these self-adjoint extension parameters may vary arbitrarily from point to point of the boundary surface. However, in the context of the Casimir effect, such a generality seems to be excessive, lacking physical motivation and, moreover, being impermissible, as long as boundary condition (51)-(52) is to be regarded as the one determining the spectrum of the wave number vector in the $z$-direction. Therefore, we shall assume in the following that the self-adjoint extension parameters are independent of coordinates $x$ and $y$.

The equation determining the spectrum of $k_{l}$, see (58), can be presented in the form

$$
\mathrm{e}^{2 \mathrm{i} k_{l} a}=\mathrm{e}^{-2 \mathrm{i} \eta_{k_{l}}}
$$

or

$$
\sin \left(k_{l} a+\eta_{k_{l}}\right)=0
$$


where

$$
\begin{gathered}
\eta_{k_{l}}=-\frac{1}{2} \arctan \left\{k_{l} \sin \tilde{\varphi}_{+}\left[m \cos \varphi_{+}-\omega_{n l} \operatorname{sgn}\left(E_{n l}\right) \cos \tilde{\varphi}_{+}\right]^{-1}\right\} \\
-\frac{1}{2} \arctan \left\{k_{l} \sin \tilde{\varphi}_{-}\left[\left(m \cos \varphi_{-}-\omega_{n l} \operatorname{sgn}\left(E_{n l}\right) \cos \tilde{\varphi}_{-}\right]^{-1}\right\}\right. \\
\mp \frac{1}{2} \arctan \left(k _ { l } \left\{m ^ { 2 } \left[2-2 \cos \left(\varphi_{+}-\varphi_{-}\right)\left(\cos \varphi_{+} \cos \varphi_{-}+\cos \tilde{\varphi}_{+} \cos \tilde{\varphi}_{-}\right)\right.\right.\right. \\
\left.+2 \cos \varphi_{+} \cos \varphi_{-} \cos \tilde{\varphi}_{+} \cos \tilde{\varphi}_{-}-\sin ^{2} \varphi_{+} \sin ^{2} \tilde{\varphi}_{-}-\sin ^{2} \varphi_{-} \sin ^{2} \tilde{\varphi}_{+}\right] \\
-2 m \omega_{n l} \operatorname{sgn}\left(E_{n l}\right)\left[\cos \varphi_{+} \cos \tilde{\varphi}_{+}+\cos \varphi_{-} \cos \tilde{\varphi}_{-}\right. \\
\left.-\cos \left(\varphi_{+}-\varphi_{-}\right)\left(\cos \varphi_{+} \cos \tilde{\varphi}_{-}+\cos \varphi_{-} \cos \tilde{\varphi}_{+}\right)\right] \\
-2 n|e B|\left[2 \cos \left(\varphi_{+}-\varphi_{-}\right) \cos \tilde{\varphi}_{+} \cos \tilde{\varphi}_{-}-\cos { }^{2} \tilde{\varphi}_{+}-\cos \tilde{\varphi}_{-}\right] \\
\left.+k_{l}^{2} \sin ^{2}\left(\varphi_{+}-\varphi_{-}\right)\right\}^{1 / 2}\left[m^{2}\left(\cos \varphi_{+} \cos \varphi_{-}+\cos \tilde{\varphi}_{+} \cos \tilde{\varphi}_{-}\right)\right. \\
+m \omega_{n l} \operatorname{sgn}\left(E_{n l}\right)\left(\cos \varphi_{+} \cos \tilde{\varphi}_{-}+\cos \varphi_{-} \cos \tilde{\varphi}_{+}\right)+2 n|e B| \cos \tilde{\varphi}_{+} \cos \tilde{\varphi}_{-} \\
\left.+k_{l}^{2} \cos \left(\varphi_{+}-\varphi_{-}\right){ }^{-1}\right)
\end{gathered}
$$

(two signs correspond to two roots of the quadratic equation for variable $\mathrm{e}^{2 \mathrm{i} k_{l} a}$, see (58) and (59)) and

$$
\begin{aligned}
& \eta_{k_{l}}= \arctan \left(k_{l} \sin \left[\frac{1}{2}\left(\varphi_{\operatorname{sgn}(e B)}-\varphi_{-\operatorname{sgn}(e B)}-\tilde{\varphi}_{\operatorname{sgn}(e B)}-\tilde{\varphi}_{-\operatorname{sgn}(e B)}\right)\right]\right. \\
& \times\left\{m \cos \left[\frac{1}{2}\left(\varphi_{\operatorname{sgn}(e B)}+\varphi_{-\operatorname{sgn}(e B)}-\tilde{\varphi}_{\operatorname{sgn}(e B)}+\tilde{\varphi}_{-\operatorname{sgn}(e B)}\right)\right]\right. \\
&\left.\left.-\omega_{0 l} \operatorname{sgn}\left(E_{0 l}\right) \cos \left[\frac{1}{2}\left(\varphi_{\operatorname{sgn}(e B)}-\varphi_{-\operatorname{sgn}(e B)}-\tilde{\varphi}_{\operatorname{sgn}(e B)}-\tilde{\varphi}_{-\operatorname{sgn}(e B)}\right)\right]\right\}^{-1}\right), \quad n=0 .
\end{aligned}
$$


It should be emphasized that value $k_{l}=0$ is allowed for special cases only. Really, we have in the case of $k_{l}=0$ :

$$
\left.\psi_{q n l}^{(j)}(\mathbf{r})\right|_{z=a / 2}=\left.\psi_{q n l}^{(j)}(\mathbf{r})\right|_{z=-a / 2},
$$

and boundary condition (51)-(52) can be presented in the form

$$
\left.R \psi_{q n l}^{(j)}(\mathbf{r})\right|_{k_{l}=0}=0,
$$

where

$$
\left\{\begin{array}{ll}
R_{11}=\sin \frac{\varphi_{+}-\tilde{\varphi}_{+}}{2}, \quad R_{12}=0, & R_{13}=\mathrm{i} \cos \frac{\varphi_{+}-\tilde{\varphi}_{+}}{2}, \quad R_{14}=0, \\
R_{21}=0, \quad R_{22}=\sin \frac{\varphi_{+}+\tilde{\varphi}_{+}}{2}, & R_{23}=0, \quad R_{24}=\mathrm{i} \cos \frac{\varphi_{+}+\tilde{\varphi}_{+}}{2}, \\
R_{31}=\sin \frac{\varphi_{-}+\tilde{\varphi}_{-}}{2}, \quad R_{32}=0, & R_{33}=\mathrm{i} \cos \frac{\varphi_{-}+\tilde{\varphi}_{-}}{2}, \quad R_{34}=0 \\
R_{41}=0, \quad R_{42}=\sin \frac{\varphi_{-}-\tilde{\varphi}_{-}}{2}, & R_{43}=0, \quad R_{44}=\mathrm{i} \cos \frac{\varphi_{-}-\tilde{\varphi}_{-}}{2}
\end{array}\right\} .
$$

The determinant of matrix $R$ is:

$$
\operatorname{det} R=-\sin \frac{\varphi_{+}-\varphi_{-}+\tilde{\varphi}_{+}+\tilde{\varphi}_{-}}{2} \sin \frac{\varphi_{+}-\varphi_{-}-\tilde{\varphi}_{+}-\tilde{\varphi}_{-}}{2} .
$$

The necessary and sufficient condition for value $k_{l}=0$ to be admissible is $\operatorname{det} R=0$, i.e. either

$$
\varphi_{+}-\varphi_{-}=\tilde{\varphi}_{+}+\tilde{\varphi}_{-}
$$

or

$$
\varphi_{+}-\varphi_{-}=-\tilde{\varphi}_{+}-\tilde{\varphi}_{-} .
$$

Otherwise, $\operatorname{det} R \neq 0$ and value $k_{l}=0$ is excluded from the spectrum, because equation (73) then allows for the trivial solution only, $\left.\psi_{q n l}^{(j)}(\mathbf{r})\right|_{k_{l}=0} \equiv 0$.

It is not clear which of the signs in (70) should be chosen. Moreover, the square root in the argument of the last arctangent in (70) may become imaginary for some values of the self-adjoint extension parameters, and this results in the complex values of $k_{l}$. Both of these obstructions are clearly eliminated by imposing restriction

$$
\varphi_{+}=\varphi_{-}=\varphi, \quad \tilde{\varphi}_{+}=\tilde{\varphi}_{-}=\tilde{\varphi} .
$$

Then (70) and (71) take form

$$
\eta_{k_{l}}=-\arctan \left[k_{l} \sin \tilde{\varphi}\left(m \cos \varphi-\operatorname{sgn}\left(E_{n l}\right) \omega_{n l} \cos \tilde{\varphi}\right)^{-1}\right], \quad n \geq 0,
$$


and the equation determining the spectrum of $k_{l}$ can be presented as

$$
\cos \left(k_{l} a\right)+\frac{\omega_{n l} \operatorname{sgn}\left(E_{n l}\right) \cos \tilde{\varphi}-m \cos \varphi}{k_{l} \sin \tilde{\varphi}} \sin \left(k_{l} a\right)=0 ;
$$

note that the spectrum consists of values of the same sign, say, $k_{l}>0$ (values of the opposite sign $\left(k_{l}<0\right)$ should be excluded to avoid double counting). Relations (37) and (39) in the case of (78) take forms

$$
\left.\left\{I-\beta \exp \left[\mathrm{i}\left(\varphi \gamma^{5} \pm \tilde{\varphi} \alpha^{z}\right)\right]\right\} \chi\right|_{z= \pm a / 2}=0
$$

and

$$
\left.\tilde{\chi}^{\dagger} \beta \exp \left\{\mathrm{i}\left[\varphi \gamma^{5} \pm(\tilde{\varphi}+\pi / 2) \alpha^{z}\right]\right\} \chi\right|_{z= \pm a / 2}=0 .
$$

respectively.

In the case of $\tilde{\varphi}=-\pi / 2$, the spectrum of $k_{l}$ is independent of the number of the Landau level, $n$, and of the sign of the one-particle energy, $\operatorname{sgn}\left(E_{n l}\right)$; it is determined from equation

$$
\cos \left(k_{l} a\right)+\frac{m \cos \varphi}{k_{l}} \sin \left(k_{l} a\right)=0 .
$$

In the case of $\tilde{\varphi}=0$, the $k_{l}$-spectrum is also independent of $n$ and of $\operatorname{sgn}\left(E_{n l}\right)$; moreover, it is independent of $\varphi$, since the determining equation takes form

$$
\sin \left(k_{l} a\right)=0
$$

note that value $k_{l}=0$ is admissible in this case, see (76)-(78). In what follows, we shall consider the most general case of two self-adjoint extension parameters, $\varphi$ and $\tilde{\varphi}$, when the $k_{l}$-spectrum depends on $n$ and on $\operatorname{sgn}\left(E_{n l}\right)$, see (80).

As was already mentioned, the expression for the induced vacuum energy per unit area of the boundary surface, see (66), can be regarded as purely formal, since it is ill-defined due to the divergence of infinite sums over $l$ and $n$. To tame the divergence, a factor containing a regularization parameter should be inserted in (66). A summation over values $k_{l} \geq 0$, which are determined by (80), can be performed with the use of the Abel-Plana formula and its generalizations. In the cases of $\tilde{\varphi}=0$ and of $\varphi=-\tilde{\varphi}=\pi / 2$, the well-known versions of the Abel-Plana formula (see, e.g., [17]), 


$$
\begin{gathered}
\left.\sum_{\operatorname{sgn}\left(E_{n l}\right)} \sum_{k_{l} \geq 0} f\left(k_{l}^{2}\right)\right|_{\sin \left(k_{l} a\right)=0}=\frac{a}{\pi} \int_{-\infty}^{\infty} \mathrm{d} k f\left(k^{2}\right)-\frac{2 \mathrm{i} a}{\pi} \int_{0}^{\infty} \mathrm{d} \kappa \frac{f\left[(-\mathrm{i} \kappa)^{2}\right]-f\left[(\mathrm{i} \kappa)^{2}\right]}{\mathrm{e}^{2 \kappa a}-1} \\
+f(0)
\end{gathered}
$$

and

$$
\left.\sum_{\operatorname{sgn}\left(E_{n l}\right)} \sum_{k_{l}>0} f\left(k_{l}^{2}\right)\right|_{\cos \left(k_{l} a\right)=0}=\frac{a}{\pi} \int_{-\infty}^{\infty} \mathrm{d} k f\left(k^{2}\right)+\frac{2 \mathrm{i} a}{\pi} \int_{0}^{\infty} \mathrm{d} \kappa \frac{f\left[(-\mathrm{i} \kappa)^{2}\right]-f\left[(\mathrm{i} \kappa)^{2}\right]}{\mathrm{e}^{2 \kappa a}+1},
$$

are used, respectively. Otherwise, we use the version of the Abel-Plana formula, that is derived in Appendix B:

$$
\begin{gathered}
\sum_{\operatorname{sgn}\left(E_{n l}\right)} \sum_{k_{l}>0} f\left(k_{l}^{2}\right)=\frac{a}{\pi} \int_{-\infty}^{\infty} \mathrm{d} k f\left(k^{2}\right)+\frac{2 \mathrm{i} a}{\pi} \int_{0}^{\infty} \mathrm{d} \kappa \Lambda(\kappa)\left\{f\left[(-\mathrm{i} \kappa)^{2}\right]-f\left[(\mathrm{i} \kappa)^{2}\right]\right\} \\
-f(0)-\frac{1}{\pi} \int_{-\infty}^{\infty} \mathrm{d} k f\left(k^{2}\right) \frac{m \cos \varphi \sin \tilde{\varphi}\left[k^{2}-\mu_{n}(\varphi, \tilde{\varphi})\right]}{\left[k^{2}+\mu_{n}(\varphi, \tilde{\varphi})\right]^{2}+4 k^{2} m^{2} \cos ^{2} \varphi \sin ^{2} \tilde{\varphi}}
\end{gathered}
$$

where

$$
\begin{gathered}
\Lambda(\kappa)=\left(-\left[\kappa^{2} \cos 2 \tilde{\varphi}-\mu_{n}(\varphi, \tilde{\varphi})\right] \mathrm{e}^{2 \kappa a}+\kappa^{2}+2 \kappa m \cos \varphi \sin \tilde{\varphi}-\mu_{n}(\varphi, \tilde{\varphi})\right. \\
+\frac{\sin \tilde{\varphi}}{a}\left\{-\kappa^{2} m \cos \varphi\left(\cos 2 \tilde{\varphi} \mathrm{e}^{2 \kappa a}-1\right)+\left[(2 \kappa \sin \tilde{\varphi}-m \cos \varphi) \mathrm{e}^{2 \kappa a}\right.\right. \\
\left.\left.+m \cos \varphi] \mu_{n}(\varphi, \tilde{\varphi})\right\}\left[\kappa^{2}-2 \kappa m \cos \varphi \sin \tilde{\varphi}-\mu_{n}(\varphi, \tilde{\varphi})\right]^{-1}\right) \\
\quad \times\left\{\left[\kappa^{2}-2 \kappa m \cos \varphi \sin \tilde{\varphi}-\mu_{n}(\varphi, \tilde{\varphi})\right] \mathrm{e}^{4 \kappa a}\right. \\
\left.-2\left[\kappa^{2} \cos 2 \tilde{\varphi}-\mu_{n}(\varphi, \tilde{\varphi})\right] \mathrm{e}^{2 \kappa a}+\kappa^{2}+2 \kappa m \cos \varphi \sin \tilde{\varphi}-\mu_{n}(\varphi, \tilde{\varphi})\right\}^{-1}
\end{gathered}
$$

and

$$
\mu_{n}(\varphi, \tilde{\varphi})=2 n|e B| \cos ^{2} \tilde{\varphi}+m^{2} \sin (\varphi+\tilde{\varphi}) \sin (\varphi-\tilde{\varphi})
$$


In (85)-(87), $f\left(u^{2}\right)$ as a function of complex variable $u$ is assumed to decrease sufficiently fast at large distances from the origin of the complex $u$-plane, and this decrease is due to the use of some kind of regularization for (66). However, the regularization in the second integral on the right-hand side of (85)-(87) can be removed; then

$$
\mathrm{i}\left\{f\left[(-\mathrm{i} \kappa)^{2}\right]-f\left[(\mathrm{i} \kappa)^{2}\right]\right\}=-\frac{|e B|}{\pi} \sum_{n=0}^{\infty} \iota_{n} \sqrt{\kappa^{2}-\omega_{n 0}^{2}}
$$

with the range of $\kappa$ restricted to $\kappa>\omega_{n 0}$ for the corresponding terms; here, recalling (47), $\omega_{n 0}=\sqrt{2 n|e B|+m^{2}}$. As to the first integral on the righthand side of (85)-(87), one immediately recognizes that it is equal to $\varepsilon^{\infty}$ (48) multiplied by $a$. Hence, if one ignores for a moment the last term of (85), as well as the terms in the last line of (87), then the problem of regularization and removal of the divergency in expression (66) is the same as that in the case of no boundaries, when the magnetic field fills the whole space. Defining the Casimir energy as the vacuum energy per unit area of the boundary surface, which is renormalized in the same way as in the case of no boundaries, we obtain

$$
\begin{aligned}
& \frac{E_{\mathrm{ren}}}{S}=a \varepsilon_{\mathrm{ren}}^{\infty}-\frac{2|e B|}{\pi^{2}} a \sum_{n=0}^{\infty} \iota_{n} \int_{\omega_{n 0}}^{\infty} \mathrm{d} \kappa \Lambda(\kappa) \sqrt{\kappa^{2}-\omega_{n 0}^{2}} \\
&+\frac{|e B|}{2 \pi} \sum_{n=0}^{\infty} \iota_{n} \omega_{n 0}+\frac{|e B|}{2 \pi^{2}} \int_{-\infty}^{\infty} \mathrm{d} k \sum_{n=0}^{\infty} \iota_{n} \sqrt{k^{2}+\omega_{n 0}^{2}} \\
& \quad \times \frac{m \cos \varphi \sin \tilde{\varphi}\left[k^{2}-\mu_{n}(\varphi, \tilde{\varphi})\right]}{\left[k^{2}+\mu_{n}(\varphi, \tilde{\varphi})\right]^{2}+4 k^{2} m^{2} \cos ^{2} \varphi \sin ^{2} \tilde{\varphi}}
\end{aligned}
$$

$\varepsilon_{\text {ren }}^{\infty}$ is given by (49). The sums and the integral in the last two lines of (90) (which are due to the terms in the last line of (87) and which can be interpreted as describing the proper energies of the boundary planes containing the sources of the magnetic field) are divergent, but this divergency is of no concern for us, because it has no physical consequences. Rather than the Casimir energy, a physically relevant quantity is the Casimir force per unit area of the boundary surface, i.e. pressure, which is defined as

$$
F=-\frac{\partial}{\partial a} \frac{E_{\mathrm{ren}}}{S}
$$


and which is free from divergencies. We obtain

$$
F=-\varepsilon_{\text {ren }}^{\infty}-\frac{2|e B|}{\pi^{2}} \sum_{n=0}^{\infty} \iota_{n} \int_{\omega_{n 0}}^{\infty} \mathrm{d} \kappa \Upsilon(\kappa) \sqrt{\kappa^{2}-\omega_{n 0}^{2}}
$$

where

$$
\begin{gathered}
\Upsilon(\kappa) \equiv-\frac{\partial}{\partial a} a \Lambda(\kappa)=\left[v_{1}(\kappa) \mathrm{e}^{6 \kappa a}+v_{2}(\kappa) \mathrm{e}^{4 \kappa a}+v_{3}(\kappa) \mathrm{e}^{2 \kappa a}+v_{4}(\kappa)\right] \\
\times\left\{\left[\kappa^{2}-2 \kappa m \cos \varphi \sin \tilde{\varphi}-\mu_{n}(\varphi, \tilde{\varphi})\right] \mathrm{e}^{4 \kappa a}\right. \\
\left.-2\left[\kappa^{2} \cos 2 \tilde{\varphi}-\mu_{n}(\varphi, \tilde{\varphi})\right] \mathrm{e}^{2 \kappa a}+\kappa^{2}+2 \kappa m \cos \varphi \sin \tilde{\varphi}-\mu_{n}(\varphi, \tilde{\varphi})\right\}^{-2}
\end{gathered}
$$

and

$$
\begin{gathered}
v_{1}(\kappa)=-(2 \kappa a-1)\left[\kappa^{2}-2 \kappa m \cos \varphi \sin \tilde{\varphi}-\mu_{n}(\varphi, \tilde{\varphi})\right]\left[\kappa^{2} \cos 2 \tilde{\varphi}-\mu_{n}(\varphi, \tilde{\varphi})\right] \\
-2\left[\kappa^{2} m \cos \varphi \cos 2 \tilde{\varphi}-(2 \kappa \sin \tilde{\varphi}-m \cos \varphi) \mu_{n}(\varphi, \tilde{\varphi})\right] \kappa \sin \tilde{\varphi}, \\
v_{2}(\kappa)=(4 \kappa a-3)\left\{\left[\kappa^{2}-\mu_{n}(\varphi, \tilde{\varphi})\right]^{2}-4 \kappa^{2} m^{2} \cos ^{2} \varphi \sin ^{2} \tilde{\varphi}\right\} \\
+8 \kappa^{2}\left[\kappa^{2} \cos ^{2} \tilde{\varphi}-m^{2} \cos ^{2} \varphi-\mu_{n}(\varphi, \tilde{\varphi})\right] \sin ^{2} \tilde{\varphi}+4\left[\kappa^{2}+\mu_{n}(\varphi, \tilde{\varphi})\right] \kappa m \cos \varphi \sin \tilde{\varphi}, \\
v_{3}(\kappa)=-(2 \kappa a-3)\left[\kappa^{2}+2 \kappa m \cos \varphi \sin \tilde{\varphi}-\mu_{n}(\varphi, \tilde{\varphi})\right]\left[\kappa^{2} \cos 2 \tilde{\varphi}-\mu_{n}(\varphi, \tilde{\varphi})\right] \\
-2\left[\kappa^{2} m \cos \varphi \cos 2 \tilde{\varphi}+(2 \kappa \sin \tilde{\varphi}+m \cos \varphi) \mu_{n}(\varphi, \tilde{\varphi})\right] \kappa \sin \tilde{\varphi}, \\
v_{4}(\kappa)=-\left[\kappa^{2}+2 \kappa m \cos \varphi \sin \tilde{\varphi}-\mu_{n}(\varphi, \tilde{\varphi})\right]^{2} .
\end{gathered}
$$

\section{Some particular cases}

In the cases of $\tilde{\varphi}=-\pi / 2$ and of $\tilde{\varphi}=0$, relations (93)-(97) are simplified:

$\left.\Upsilon(\kappa)\right|_{\tilde{\varphi}=-\pi / 2}=\frac{\left[(2 \kappa a-1)\left(\kappa^{2}-m^{2} \cos ^{2} \varphi\right)-2 \kappa m \cos \varphi\right] \mathrm{e}^{2 \kappa a}-(\kappa-m \cos \varphi)^{2}}{\left[(\kappa+m \cos \varphi) \mathrm{e}^{2 \kappa a}+\kappa-m \cos \varphi\right]^{2}}$

and

$$
\left.\Upsilon(\kappa)\right|_{\tilde{\varphi}=0}=-\frac{(2 \kappa a-1) \mathrm{e}^{2 \kappa a}+1}{\left(\mathrm{e}^{2 \kappa a}-1\right)^{2}}
$$


Recalling the choice of off-diagonal matrix $K(14)$ at $u^{2}-v^{2}-\boldsymbol{t}^{2}>0$, see (18), we note that boundary condition (40) under restriction

$$
\vartheta_{+}=\vartheta_{-}=\vartheta, \quad \tilde{\vartheta}_{+}=\tilde{\vartheta}_{-}=0
$$

takes form

$$
\left.\left(I \pm \mathrm{i} \beta \alpha^{z} \cosh \vartheta+\mathrm{i} \gamma^{5} \sinh \vartheta\right) \chi\right|_{z= \pm a / 2}=0 .
$$

As has been proven in [12], the appropriate equation determining the spectrum of $k_{l}$ in this case is

$$
\cos \left(k_{l} a\right)+\frac{m}{k_{l} \cosh \vartheta} \sin \left(k_{l} a\right)=0,
$$

and the Casimir pressure is given by (92) with

$$
\Upsilon(\kappa)=\frac{\left[(2 \kappa a-1)\left(\kappa^{2} \cosh ^{2} \vartheta-m^{2}\right)-2 \kappa m \cosh \vartheta\right] \mathrm{e}^{2 \kappa a}-(\kappa \cosh \vartheta-m)^{2}}{\left[(\kappa \cosh \vartheta+m) \mathrm{e}^{2 \kappa a}+\kappa \cosh \vartheta-m\right]^{2}} .
$$

Comparing (83) and (98) with (102) and (103), we see that the case of the Hermitian $K$-matrix restricted by (78) and condition $\tilde{\varphi}=-\pi / 2$ is obtainable by substitution $\cosh \vartheta \rightarrow 1 / \cos \varphi$ from the case of the off-diagonal $K$-matrix restricted by (100); the latter case was exhaustively studied in [12]. We only remind here that the case of the MIT bag boundary condition corresponds to $\varphi=0$, or, respectively, $\vartheta=0$.

In the case of $\tilde{\varphi}=0$, we obtain $k_{l}=\frac{\pi}{a} l(l=0,1,2, \ldots)$ and

$$
\left.\frac{E_{\mathrm{ren}}}{S}\right|_{\tilde{\varphi}=0}=a \varepsilon_{\text {ren }}^{\infty}+\frac{2|e B|}{\pi^{2}} a \sum_{n=0}^{\infty} \iota_{n} \int_{\omega_{n 0}}^{\infty} \mathrm{d} \kappa \frac{\sqrt{\kappa^{2}-\omega_{n 0}^{2}}}{\mathrm{e}^{2 \kappa a}-1}-\frac{|e B|}{2 \pi} \sum_{n=0}^{\infty} \iota_{n} \omega_{n 0}
$$

and

$$
\left.F\right|_{\tilde{\varphi}=0}=-\varepsilon_{\text {ren }}^{\infty}+\frac{2|e B|}{\pi^{2}} \sum_{n=0}^{\infty} \iota_{n} \int_{\omega_{n 0}}^{\infty} \frac{\mathrm{d} \kappa}{\mathrm{e}^{2 \kappa a}-1} \frac{\kappa^{2}}{\sqrt{\kappa^{2}-\omega_{n 0}^{2}}} .
$$

The integral in (104) can be taken after expanding the factor with denominator as $\sum_{j=1}^{\infty} \mathrm{e}^{-2 j \kappa a}$. In this way, we obtain the following expressions for the Casimir energy

$$
\left.\frac{E_{\mathrm{ren}}}{S}\right|_{\tilde{\varphi}=0}=a \varepsilon_{\mathrm{ren}}^{\infty}+\frac{|e B|}{\pi^{2}} \sum_{n=0}^{\infty} \iota_{n} \omega_{n 0} \sum_{j=1}^{\infty} \frac{1}{j} K_{1}\left(2 j \omega_{n 0} a\right)-\frac{|e B|}{2 \pi} \sum_{n=0}^{\infty} \iota_{n} \omega_{n 0}
$$


and the Casimir pressure

$$
\left.F\right|_{\tilde{\varphi}=0}=-\varepsilon_{\text {ren }}^{\infty}+\frac{2|e B|}{\pi^{2}} \sum_{n=0}^{\infty} \iota_{n} \omega_{n 0}^{2} \sum_{j=1}^{\infty}\left[K_{0}\left(2 j \omega_{n 0} a\right)+\frac{1}{2 j \omega_{n 0} a} K_{1}\left(2 j \omega_{n 0} a\right)\right]
$$

where $K_{\nu}(s)$ is the Macdonald function of order $\nu$.

It should be noted that the periodic boundary condition,

$$
\left.\chi\right|_{z=-a / 2}=\left.\chi\right|_{z=a / 2},\left.\quad \tilde{\chi}\right|_{z=-a / 2}=\left.\tilde{\chi}\right|_{z=a / 2},
$$

ensures the self-adjointness of the Dirac hamiltonian operator, but current $J_{q n l j}^{z}(\mathbf{r})=\psi_{q n l}^{(j) \dagger}(\mathbf{r}) \alpha^{z} \psi_{q n l}^{(j)}(\mathbf{r})(j=0,1,2)$ does not vanish at the boundary: instead, the influx of the quantized matter through a one boundary plane equals the outflux of the quantized matter through another boundary plane,

$$
\left.J_{q n l j}^{z}(\mathbf{r})\right|_{z=-a / 2}=\left.J_{q n l j}^{z}(\mathbf{r})\right|_{z=a / 2} .
$$

The spectrum of the wave number vector which is orthogonal to the boundary is $k_{l}=\frac{2 \pi}{a} l(l=0, \pm 1, \pm 2, \ldots)$, and the Casimir pressure is

$$
(F)_{\text {periodic }}=-\varepsilon_{\text {ren }}^{\infty}+\frac{2|e B|}{\pi^{2}} \sum_{n=0}^{\infty} \iota_{n} \int_{\omega_{n 0}}^{\infty} \frac{\mathrm{d} \kappa}{\mathrm{e}^{\kappa a}-1} \frac{\kappa^{2}}{\sqrt{\kappa^{2}-\omega_{n 0}^{2}}}
$$

or, in the alternative representation,

$$
(F)_{\text {periodic }}=-\varepsilon_{\text {ren }}^{\infty}+\frac{2|e B|}{\pi^{2}} \sum_{n=0}^{\infty} \iota_{n} \omega_{n 0}^{2} \sum_{j=1}^{\infty}\left[K_{0}\left(j \omega_{n 0} a\right)+\frac{1}{j \omega_{n 0} a} K_{1}\left(j \omega_{n 0} a\right)\right] \text {. }
$$

It is instructive to consider also the case of $\varphi=\pi / 2$, when relations (93)-(97) are reduced to 


$$
\begin{aligned}
\left.\Upsilon(\kappa)\right|_{\varphi=\pi / 2}=-\{ & {\left[(2 \kappa a-1)\left(1-\frac{2 \kappa^{2} \sin ^{2} \tilde{\varphi}}{\kappa^{2}-\omega_{n 0}^{2} \cos ^{2} \tilde{\varphi}}\right)-\frac{\kappa^{2} \omega_{n 0}^{2} \sin ^{2} 2 \tilde{\varphi}}{\left(\kappa^{2}-\omega_{n 0}^{2} \cos ^{2} \tilde{\varphi}\right)^{2}}\right] \mathrm{e}^{6 \kappa a} } \\
& -\left[4 \kappa a-3+\frac{2 \kappa^{2}\left(\kappa^{2}-\omega_{n 0}^{2}\right) \sin ^{2} 2 \tilde{\varphi}}{\left(\kappa^{2}-\omega_{n 0}^{2} \cos ^{2} \tilde{\varphi}\right)^{2}}\right] \mathrm{e}^{4 \kappa a} \\
+ & {\left.\left[(2 \kappa a-3)\left(1-\frac{2 \kappa^{2} \sin ^{2} \tilde{\varphi}}{\kappa^{2}-\omega_{n 0}^{2} \cos ^{2} \tilde{\varphi}}\right)+\frac{\kappa^{2} \omega_{n 0}^{2} \sin ^{2} 2 \tilde{\varphi}}{\left(\kappa^{2}-\omega_{n 0}^{2} \cos ^{2} \tilde{\varphi}\right)^{2}}\right] \mathrm{e}^{2 \kappa a}+1\right\} } \\
& \times\left[\left(\mathrm{e}^{2 \kappa a}-1\right)^{2}+\frac{4 \kappa^{2} \sin ^{2} \tilde{\varphi}}{\kappa^{2}-\omega_{n 0}^{2} \cos ^{2} \tilde{\varphi}} \mathrm{e}^{2 \kappa a}\right]^{-2} .
\end{aligned}
$$

This case interpolates between the case of spectrum $k_{l}=\frac{\pi}{a} l(l=0,1,2, \ldots)$, see (99) and (104)-(107), and the case of spectrum $k_{l}=\frac{\pi}{a}\left(l+\frac{1}{2}\right)(l=0,1,2, \ldots)$ with

$$
\left.\Upsilon(\kappa)\right|_{\varphi=-\tilde{\varphi}=\pi / 2}=\frac{(2 \kappa a-1) \mathrm{e}^{2 \kappa a}-1}{\left(\mathrm{e}^{2 \kappa a}+1\right)^{2}}
$$

and

$$
\left.F\right|_{\varphi=-\tilde{\varphi}=\pi / 2}=-\varepsilon_{\mathrm{ren}}^{\infty}-\frac{2|e B|}{\pi^{2}} \sum_{n=0}^{\infty} \iota_{n} \int_{\omega_{n 0}}^{\infty} \frac{\mathrm{d} \kappa}{\mathrm{e}^{2 \kappa a}+1} \frac{\kappa^{2}}{\sqrt{\kappa^{2}-\omega_{n 0}^{2}}}
$$

or, alternatively,

$$
\begin{gathered}
\left.F\right|_{\varphi=-\tilde{\varphi}=\pi / 2}=-\varepsilon_{\text {ren }}^{\infty} \\
-\frac{2|e B|}{\pi^{2}} \sum_{n=0}^{\infty} \iota_{n} \omega_{n 0}^{2} \sum_{j=1}^{\infty}(-1)^{j-1}\left[K_{0}\left(2 j \omega_{n 0} a\right)+\frac{1}{2 j \omega_{n 0} a} K_{1}\left(2 j \omega_{n 0} a\right)\right] .
\end{gathered}
$$

Concluding this section, let us note that in the case of spectrum $k_{l}=$ $\frac{2 \pi}{a}\left(l+\frac{1}{2}\right)(l=0, \pm 1, \pm 2, \ldots)$, corresponding to the antiperiodic boundary condition,

$$
\left.\chi\right|_{z=-a / 2}=-\left.\chi\right|_{z=a / 2},\left.\quad \tilde{\chi}\right|_{z=-a / 2}=-\left.\tilde{\chi}\right|_{z=a / 2},
$$

one obtains [12]

$$
(F)_{\text {antiperiodic }}=-\varepsilon_{\mathrm{ren}}^{\infty}-\frac{2|e B|}{\pi^{2}} \sum_{n=0}^{\infty} \iota_{n} \int_{\omega_{n 0}}^{\infty} \frac{\mathrm{d} \kappa}{\mathrm{e}^{\kappa a}+1} \frac{\kappa^{2}}{\sqrt{\kappa^{2}-\omega_{n 0}^{2}}}
$$


or, alternatively,

$$
\begin{gathered}
(F)_{\text {antiperiodic }}=-\varepsilon_{\text {ren }}^{\infty} \\
-\frac{2|e B|}{\pi^{2}} \sum_{n=0}^{\infty} \iota_{n} \omega_{n 0}^{2} \sum_{j=1}^{\infty}(-1)^{j-1}\left[K_{0}\left(j \omega_{n 0} a\right)+\frac{1}{j \omega_{n 0} a} K_{1}\left(j \omega_{n 0} a\right)\right] .
\end{gathered}
$$

\section{Asymptotics at small and large separations of plates}

The expression for the Casimir pressure, see (92), can be presented as

$$
F=-\varepsilon_{\text {ren }}^{\infty}+\Delta_{\varphi, \tilde{\varphi}}(a)
$$

where the first term is equal to minus the vacuum energy density which is induced by the magnetic field in unbounded space, see (49), whereas the second term which is given by the sum over $n$ and the integral over $\kappa$ in (92) depends on the distance between bounding plates and on a choice of boundary conditions at the plates.

In the case of a weak magnetic field, $|B| \ll m^{2}|e|^{-1}$, substituting the sum by integral $\int_{0}^{\infty} \mathrm{d} n$ and changing the integration variable, we get

$$
\Delta_{\varphi, \tilde{\varphi}}(a)=-\frac{1}{\pi^{2}} \int_{m}^{\infty} \mathrm{d} \kappa\left(\kappa^{2}-m^{2}\right)^{3 / 2} \int_{0}^{1} \mathrm{~d} v \sqrt{1-v} \tilde{\Upsilon}(\kappa, v), \quad|e B| \ll m^{2},
$$

where $\tilde{\Upsilon}(\kappa, v)$ is obtained from $\Upsilon(\kappa)(93)$ by substitution $\mu_{n}(\varphi, \tilde{\varphi}) \rightarrow \tilde{\mu}_{v, \kappa^{2}}(\varphi, \tilde{\varphi})$ with

$$
\tilde{\mu}_{v, \kappa^{2}}(\varphi, \tilde{\varphi})=v\left(\kappa^{2}-m^{2}\right) \cos ^{2} \tilde{\varphi}+m^{2} \sin (\varphi+\tilde{\varphi}) \sin (\varphi-\tilde{\varphi}) .
$$

In the limit of small distances between the plates, $m a \ll 1$, (120) becomes independent of the $\varphi$-parameter:

$$
\begin{aligned}
\Delta_{\varphi, \tilde{\varphi}}(a)= & \frac{1}{4 a^{4}}\left\{\frac{\pi^{2}}{30}-\int_{0}^{1} \mathrm{~d} v \rho_{\tilde{\varphi}}(v)\left(1-\frac{\left|\rho_{\tilde{\varphi}}(v)\right|}{\pi}\right)\left[\frac{3}{2} \sqrt{1-v} \rho_{\tilde{\varphi}}(v)\left(1-\frac{\left|\rho_{\tilde{\varphi}}(v)\right|}{\pi}\right)\right.\right. \\
& \left.\left.+\frac{v \sin 2 \tilde{\varphi}}{1-v \cos ^{2} \tilde{\varphi}}\left(\frac{1}{2}-\frac{\left|\rho_{\tilde{\varphi}}(v)\right|}{\pi}\right)\right]\right\}, \quad \sqrt{|e B|} a \ll m a \ll 1, \quad(122)
\end{aligned}
$$


where

$$
\rho_{\tilde{\varphi}}(v)=\arcsin \left(\frac{\sin \tilde{\varphi}}{\sqrt{1-v \cos ^{2} \tilde{\varphi}}}\right) .
$$

Thus, $\Delta_{\varphi, \tilde{\varphi}}(a)$ in this case is power-dependent on the distance between the plates as $a^{-4}$ with the dimensionless constant of proportionality, either positive or negative, depending on the value of the $\tilde{\varphi}$-parameter. In particular, we get

$$
\Delta_{\varphi, 0}(a)=\frac{\pi^{2}}{120} \frac{1}{a^{4}}, \quad \sqrt{|e B|} a \ll m a \ll 1
$$

and

$$
\Delta_{\varphi,-\pi / 2}(a)=-\frac{7}{8} \frac{\pi^{2}}{120} \frac{1}{a^{4}}, \quad \sqrt{|e B|} a \ll m a \ll 1 .
$$

In the limit of large distances between the plates, $m a \gg 1, \Delta_{\varphi, \tilde{\varphi}}(a)(120)$ takes form

$$
\begin{gathered}
\Delta_{\varphi, \tilde{\varphi}}(a)=\frac{2}{\pi^{2}} \int_{m}^{\infty} \mathrm{d} \kappa \kappa\left(\kappa^{2}-m^{2}\right)^{3 / 2} \mathrm{e}^{-2 \kappa a} \int_{0}^{1} \mathrm{~d} v \sqrt{1-v} \\
\times\left\{a \frac{\kappa^{2} \cos 2 \tilde{\varphi}-\tilde{\mu}_{v, \kappa^{2}}(\varphi, \tilde{\varphi})}{\kappa^{2}-2 \kappa m \cos \varphi \sin \tilde{\varphi}-\tilde{\mu}_{v, \kappa^{2}}(\varphi, \tilde{\varphi})}\right. \\
\left.-\frac{(2 \kappa \sin \tilde{\varphi}-m \cos \varphi) \tilde{\mu}_{v, \kappa^{2}}(\varphi, \tilde{\varphi})-\kappa^{2} m \cos \varphi \cos 2 \tilde{\varphi}}{\left[\kappa^{2}-2 \kappa m \cos \varphi \sin \tilde{\varphi}-\tilde{\mu}_{v, \kappa^{2}}(\varphi, \tilde{\varphi})\right]^{2}} \sin \right\}, \\
|e B| \ll m^{2}, \quad m a \gg 1 .
\end{gathered}
$$

Clearly, (126) is suppressed as $\exp (-2 m a)$. In particular, we get

$$
\Delta_{\varphi, 0}(a)=\frac{1}{2 \pi^{3 / 2}} \frac{m^{5 / 2}}{a^{3 / 2}} \mathrm{e}^{-2 m a}\left[1+O\left(\frac{1}{m a}\right)\right],|e B| \ll m^{2}, m a \gg 1
$$

and

$$
\begin{gathered}
\Delta_{\varphi,-\pi / 2}(a)=\left\{\begin{array}{c}
-\frac{3}{16 \pi^{3 / 2}} \frac{m^{3 / 2}}{a^{5 / 2}} \mathrm{e}^{-2 m a}\left[1+O\left(\frac{1}{m a}\right)\right], \quad \varphi=0 \\
-\frac{\tan ^{2}(\varphi / 2)}{2 \pi^{3 / 2}} \frac{m^{5 / 2}}{a^{3 / 2}} \mathrm{e}^{-2 m a}\left[1+O\left(\frac{1}{m a}\right)\right], \quad \varphi \neq 0
\end{array}\right\}, \\
|e B| \ll m^{2}, \quad m a \gg 1 .
\end{gathered}
$$


In the case of a strong magnetic field, $|B| \gg m^{2}|e|^{-1}$, one has

$$
\begin{gathered}
\Delta_{\varphi, \tilde{\varphi}}(a)=-\frac{|e B|}{\pi^{2}}\left[\left.\int_{m}^{\infty} \mathrm{d} \kappa \sqrt{\kappa^{2}-m^{2}} \Upsilon(\kappa)\right|_{n=0}\right. \\
\left.+\left.2 \sum_{n=1}^{\infty} \int_{\sqrt{2 n|e B|}}^{\infty} \mathrm{d} \kappa \sqrt{\kappa^{2}-2 n|e B|} \Upsilon(\kappa)\right|_{m=0}\right], \quad|e B| \gg m^{2} .
\end{gathered}
$$

In the limit of extremely small distances between the plates, $m a \ll \sqrt{|e B|} a \ll 1$, the analysis is similar to that of the limit of $\sqrt{|e B|} a \ll m a \ll 1$, yielding the same results as (122)-(125). Otherwise, in the limit of $\sqrt{|e B|} a \gg 1$, only the first term in square brackets on the right-hand side of (129) matters. In the limit of small distances between the plates this term becomes $\varphi$-independent, yielding

$$
\Delta_{\varphi, \tilde{\varphi}}(a)=\frac{|e B|}{4 a^{2}}\left[\frac{1}{6}-\frac{|\tilde{\varphi}|}{\pi}\left(1-\frac{|\tilde{\varphi}|}{\pi}\right)\right], \quad \sqrt{|e B|} a \gg 1, m a \ll 1 .
$$

In particular, we get

$$
\begin{gathered}
\Delta_{\varphi, 0}(a)=\frac{|e B|}{24 a^{2}}, \quad \sqrt{|e B|} a \gg 1, m a \ll 1, \\
\Delta_{\varphi, \pm \pi / 4}(a)=-\frac{|e B|}{192 a^{2}}, \quad \sqrt{|e B|} a \gg 1, m a \ll 1
\end{gathered}
$$

and

$$
\Delta_{\varphi,-\pi / 2}(a)=-\frac{|e B|}{48 a^{2}}, \quad \sqrt{|e B|} a \gg 1, m a \ll 1 .
$$

In the limit of large distances between the plates, the first term in square brackets on the right-hand side of (129) yields 


$$
\begin{gathered}
\Delta_{\varphi, \tilde{\varphi}}(a)=\frac{2|e B|}{\pi^{2}} \int_{m}^{\infty} \mathrm{d} \kappa \kappa\left(\kappa^{2}-m^{2}\right)^{1 / 2} \mathrm{e}^{-2 \kappa a} \\
\times\left\{a \frac{\kappa^{2} \cos 2 \tilde{\varphi}-m^{2} \sin (\varphi+\tilde{\varphi}) \sin (\varphi-\tilde{\varphi})}{\kappa^{2}-2 \kappa m \cos \varphi \sin \tilde{\varphi}-m^{2} \sin (\varphi+\tilde{\varphi}) \sin (\varphi-\tilde{\varphi})}\right. \\
\left.+\frac{\kappa^{2} m \cos \varphi \cos 2 \tilde{\varphi}-(2 \kappa \sin \tilde{\varphi}-m \cos \varphi) m^{2} \sin (\varphi+\tilde{\varphi}) \sin (\varphi-\tilde{\varphi})}{\left[\kappa^{2}-2 \kappa m \cos \varphi \sin \tilde{\varphi}-m^{2} \sin (\varphi+\tilde{\varphi}) \sin (\varphi-\tilde{\varphi})\right]^{2}} \sin \right\}, \\
\sqrt{|e B|} a \gg m a \gg 1,
\end{gathered}
$$

which is obviously suppressed as $\exp (-2 m a)$. In particular, we get

$$
\Delta_{\varphi, 0}(a)=\frac{|e B|}{2 \pi^{3 / 2}} \frac{m^{3 / 2}}{a^{1 / 2}} \mathrm{e}^{-2 m a}\left[1+O\left(\frac{1}{m a}\right)\right], \quad \sqrt{|e B|} a \gg m a \gg 1
$$

and

$$
\Delta_{\varphi,-\pi / 2}(a)=\left\{\begin{array}{c}
-\frac{|e B|}{16 \pi^{3 / 2}} \frac{m^{1 / 2}}{a^{3 / 2}} \mathrm{e}^{-2 m a}\left[1+O\left(\frac{1}{m a}\right)\right], \quad \varphi=0 \\
-\frac{|e B| \tan ^{2}(\varphi / 2)}{2 \pi^{3 / 2}} \frac{m^{3 / 2}}{a^{1 / 2}} \mathrm{e}^{-2 m a}\left[1+O\left(\frac{1}{m a}\right)\right], \quad \varphi \neq 0
\end{array}\right\},
$$

It is appropriate in this section to consider also the limiting case of $m \rightarrow 0$. In view of the asymptotical behaviour of the boundary-independent piece of the Casimir pressure,

$$
-\varepsilon_{\text {ren }}^{\infty}=\frac{e^{2} B^{2}}{24 \pi^{2}} \ln \frac{2|e B|}{m^{2}}, \quad m^{2} \ll|e B|
$$

and

$$
-\varepsilon_{\mathrm{ren}}^{\infty}=\frac{1}{360 \pi^{2}} \frac{e^{4} B^{4}}{m^{4}}, \quad m^{2} \gg|e B|,
$$

namely asymptotics (122) is relevant for this case, and the pressure from the vacuum of a confined massless spinor matter field is given by expression 


$$
\begin{gathered}
\left.F\right|_{m=0, B=0}=\frac{1}{8 a^{4}}\left\{\frac{\pi^{2}}{30}-\int_{0}^{1} \mathrm{~d} v \rho_{\tilde{\varphi}}(v)\left(1-\frac{\left|\rho_{\tilde{\varphi}}(v)\right|}{\pi}\right)\right. \\
\left.\times\left[\frac{3}{2} \sqrt{1-v} \rho_{\tilde{\varphi}}(v)\left(1-\frac{\left|\rho_{\tilde{\varphi}}(v)\right|}{\pi}\right)+\frac{v \sin 2 \tilde{\varphi}}{1-v \cos ^{2} \tilde{\varphi}}\left(\frac{1}{2}-\frac{\left|\rho_{\tilde{\varphi}}(v)\right|}{\pi}\right)\right]\right\},
\end{gathered}
$$

which is bounded from above and below by values

$$
\left.F\right|_{m=0, B=0, \tilde{\varphi}=0}=\frac{\pi^{2}}{240} \frac{1}{a^{4}}
$$

and

$$
\left.F\right|_{m=0, B=0, \tilde{\varphi}=-\pi / 2}=-\frac{7}{8} \frac{\pi^{2}}{240} \frac{1}{a^{4}},
$$

respectively; here, an additional factor of $1 / 2$ has appeared due to diminishment of the number of degrees of freedom (a massless spinor can be either left or right).

\section{Summary and discussion}

In the present paper, we consider an impact of a background (classical) magnetic field on the vacuum of a quantized charged spinor matter field which is confined to a bounded region of space; the sources of the magnetic field are outside of the bounded region, and the magnetic field strength lines are assumed to be orthogonal to a boundary. The confinement of the matter field (i.e. absence of the matter flux across the boundary) is ensured by boundary condition (34) which is compatible with the self-adjointness of the Dirac hamiltonan operator and which generalizes the well-known MIT bag boundary condition to the most extent; the parameters of this general boundary condition can be interpreted as the self-adjoint extension parameters. In the case which is relevant to the geometry of the Casimir effect (i.e. the spatial region bounded by two parallel planes separated by distance $a$ ) and the uniform magnetic field orthogonal to the planes, the eight-parameter boundary condition is reduced to the four-parameter one, see (37). With the use of the latter condition we obtain the equation determining the spectrum of the wave number vector along the magnetic field, see (69)-(71). Following the analysis of this equation, we finally arrive at the two-parameter boundary 
condition, see (81), as the most general extension of the MIT bag boundary condition in the context of the Casimir effect. The spectrum of the wave number vector along the magnetic field in this case depends on the number of the Landau level and on the sign of the one-particle energy, see (80). The Casimir pressure is shown to take form of (92), where $\varepsilon_{\text {ren }}^{\infty}$ is given by (49) and $\Upsilon(\kappa)$ is given by (93)-(97). The result for the case of the MIT bag boundary condition is obtained from $(92)$ at $\varphi=0, \tilde{\varphi}=-\pi / 2$. It should be noted that the periodic and antiperiodic boundary conditions, see (108) and (116), do not ensure the confinement of the matter field: instead, they correspond to the equality between the matter influx through a one boundary plane and the matter outflux through another boundary plane, see (109). Nevertheless, the Casimir pressure in these two cases is obtainable by substitution $a \rightarrow a / 2$ in (92) at $\tilde{\varphi}=0$ (periodic boundary condition) and at $\varphi=-\tilde{\varphi}=\pi / 2$ (antiperiodic boundary condition), see (110)-(111) and (117)-(118), respectively.

The Casimir effect is usually validated in experiments with nearly parallel plates separated by a distance of order $10^{-8}-10^{-5} \mathrm{~m}$, see, e.g., [17]. The Compton wavelength of the lightest charged particle, electron, is $m^{-1} \sim$ $10^{-12} \mathrm{~m}$, thus $\mathrm{ma} \gg 1$ and, as has been shown in the preceding section, all the dependence of the Casimir pressure on the distance between the plates and a choice of boundary conditions at the plates is suppressed by factor $\exp (-2 m a)$, see (126)-(128) and (134)-(136). Hence, the pressure from the electron-positron vacuum onto the plates separated by distance $a>10^{-10} \mathrm{~m}$ is well approximated by $F \approx-\varepsilon_{\text {ren }}^{\infty}$, where $\varepsilon_{\text {ren }}^{\infty}(49)$ is negative, i.e. the pressure is positive and the plates are repelled. Some possibilities to detect this new-type Casimir effect were pointed out in [12].

Let us also discuss an application of our results to hadron physics. Since the hadron size (confinement radius) is $a \sim 10^{-15} \mathrm{~m}$ and the Compton wavelength of the lightest quark is $m^{-1} \sim 10^{-13} \mathrm{~m}$, it looks likely that asymptotical regime $m a \ll 1$ might be relevant. Although the geometry of compact bounded space (for instance, of a sphere) is more appropriate to this case, we would like just to emphasize here that the behaviour of the pressure from the vacuum of the confined quark-antiquark matter differs drastically for weak and strong magnetic fields; this has been shown in the present paper for the geometry of noncompact space bounded by two parallel planes and the uniform magnetic field orthogonal to the planes. If $-\varepsilon_{\text {ren }}^{\infty} \ll\left|\Delta_{\varphi, \tilde{\varphi}}(a)\right|$, then the pressure is $F \approx \Delta_{\varphi, \tilde{\varphi}}(a)$ which can be either positive or negative, depending on boundary conditions and independent of the magnetic field strength, see 
(122)-(125); this case 11 is relevant for $|B|<10^{13}$ Gauss. If $-\varepsilon_{\text {ren }}^{\infty} \gg\left|\Delta_{\varphi, \tilde{\varphi}}(a)\right|$, then the pressure is $F \approx-\varepsilon_{\text {ren }}^{\infty}$ which is positive and independent of boundary conditions, see (137); this case is relevant for $|B|>10^{19}$ Gauss. The pressure for the intermediate magnetic fields, $10^{13}$ Gauss $<|B|<10^{19}$ Gauss, depends both on boundary conditions and on the magnetic field strength. The magnetic fields of strength up to $10^{17}-10^{18}$ Gauss may exist in some compact astrophysical objects (magnetars) [18], while even much stronger magnetic fields are supposed to have existed in early universe [19].

\section{Acknowledgments}

We acknowledge the support from the National Academy of Sciences of Ukraine (project No.0112U000054). The work of Yu. A. S. was supported by the Program of Fundamental Research of the Department of Physics and Astronomy of the National Academy of Sciences of Ukraine (project No.0112U000056) and by the ICTP - SEENET-MTP grant PRJ-09 "Strings and Cosmology".

\section{Appendix A. Solution to the Dirac equation in a background uniform magnetic field}

A solution to the Dirac equation in the background of a static uniform magnetic field is well-described in the literature, see, e.g., 20]. Taking $e B>0$ for definiteness, the solution with positive energy is

$$
\begin{aligned}
\left.\psi_{q n k}(\mathbf{r})\right|_{E_{n k}=\omega_{n k}} & =\frac{\mathrm{e}^{\mathrm{i} q x} \mathrm{e}^{\mathrm{i} k z}}{2 \pi \sqrt{2 \omega_{n k}\left(\omega_{n k}+m\right)}}\left[C_{1}\left(\begin{array}{c}
\left(\omega_{n k}+m\right) Y_{n}^{q}(y) \\
0 \\
k Y_{n}^{q}(y) \\
\sqrt{2 n e B Y_{n-1}^{q}(y)}
\end{array}\right)\right. \\
& \left.+C_{2}\left(\begin{array}{c}
\left(\omega_{n k}+m\right) Y_{n-1}^{q}(y) \\
\sqrt{2 n e B} Y_{n}^{q}(y) \\
-k Y_{n-1}^{q}(y)
\end{array}\right)\right], \quad n \geq 1
\end{aligned}
$$

\footnotetext{
${ }^{1}$ Note that the pressure equals twice the pressure from the vacuum of the confined massless neutral spinor matter, see (139).
} 
and

$$
\left.\psi_{q 0 k}^{(0)}(\mathbf{r})\right|_{E_{0 k}=\omega_{0 k}}=\frac{\mathrm{e}^{\mathrm{i} q x} \mathrm{e}^{\mathrm{i} k z}}{2 \pi \sqrt{2 \omega_{0 k}\left(\omega_{0 k}+m\right)}} C_{0} Y_{0}^{q}(y)\left(\begin{array}{c}
\omega_{0 k}+m \\
0 \\
k \\
0
\end{array}\right),
$$

while the solution with negative energy is

$$
\begin{aligned}
\left.\psi_{q n k}(\mathbf{r})\right|_{E_{n k}=-\omega_{n k}} & =\frac{\mathrm{e}^{-\mathrm{i} q x} \mathrm{e}^{-\mathrm{i} k z}}{2 \pi \sqrt{2 \omega_{n k}\left(\omega_{n k}+m\right)}}\left[\tilde{C}_{1}\left(\begin{array}{c}
k Y_{n}^{-q}(y) \\
-\sqrt{2 n e B} Y_{n-1}^{-q}(y) \\
\left(\omega_{n k}+m\right) Y_{n}^{-q}(y) \\
0
\end{array}\right)\right. \\
& \left.+\tilde{C}_{2}\left(\begin{array}{c}
-\sqrt{2 n e B} Y_{n}^{-q}(y) \\
-k Y_{n-1}^{-q}(y) \\
0 \\
\left(\omega_{n k}+m\right) Y_{n-1}^{-q}(y)
\end{array}\right)\right], \quad n \geq 1
\end{aligned}
$$

and

$$
\left.\psi_{q 0 k}^{(0)}(\mathbf{r})\right|_{E_{0 k}=-\omega_{0 k}}=\frac{\mathrm{e}^{-\mathrm{i} q x} \mathrm{e}^{-\mathrm{i} k z}}{2 \pi \sqrt{2 \omega_{0 k}\left(\omega_{0 k}+m\right)}} \tilde{C}_{0} Y_{0}^{-q}(y)\left(\begin{array}{c}
k \\
0 \\
\omega_{0 k}+m \\
0
\end{array}\right)
$$

here $-\infty<q<\infty$ and

$$
Y_{n}^{q}(y)=\sqrt{\frac{(e B)^{1 / 2}}{2^{n} n ! \pi^{1 / 2}}} \exp \left[-\frac{e B}{2}\left(y+\frac{q}{e B}\right)^{2}\right] H_{n}\left[\sqrt{e B}\left(y+\frac{q}{e B}\right)\right]
$$

$H_{n}(v)=(-1)^{n} \mathrm{e}^{v^{2}} \frac{\mathrm{d}^{n}}{\mathrm{~d} v^{n}} \mathrm{e}^{-v^{2}}$ is the Hermite polynomial. The case of $e B<0$ is obtained by charge conjugation, i.e. changing $e B \rightarrow-e B$ and multiplying the complex conjugates of the previous solutions by i $\beta \alpha^{2}$ (the energy sign is changed to the opposite).

Solutions with different signs of energy are orthogonal:

$$
\left.\left.\int \mathrm{d}^{3} r \psi_{-q n-k}^{(j) \dagger}(\mathbf{r})\right|_{E_{n k}=-\omega_{n k}} \psi_{q^{\prime} n^{\prime} k^{\prime}}^{\left(j^{\prime}\right)}(\mathbf{r})\right|_{E_{n^{\prime} k^{\prime}}=\omega_{n^{\prime} k^{\prime}}}=0 .
$$


In the case of $n \geq 1$, two linearly independent solutions with, say, positive energy, $\left.\psi_{q n k}^{(1)}(\mathbf{r})\right|_{E_{n k}=\omega_{n k}}$ and $\left.\psi_{q n k}^{(2)}(\mathbf{r})\right|_{E_{n k}=\omega_{n k}}$, are orthogonal, if the appropriate coefficients, $C_{j}^{(1)}$ and $C_{j}^{(2)}(j=1,2)$, obey condition

$$
\sum_{j=1,2} C_{j}^{(1) *} C_{j}^{(2)}=0
$$

We impose further condition

$$
\sum_{j=1,2}\left|C_{j}^{\left(j^{\prime}\right)}\right|^{2}=\left|C_{0}\right|^{2}=1, \quad j^{\prime}=1,2 .
$$

The same conditions are demanded for coefficients $\tilde{C}_{0}$ and $\tilde{C}_{j}^{\left(j^{\prime}\right)} \quad\left(j, j^{\prime}=1,2\right)$ corresponding to the case of the negative-energy solutions. Then the wave functions satisfy the requirements of orthonormality

$$
\int \mathrm{d}^{3} r \psi_{q n k}^{(j) \dagger}(\mathbf{r}) \psi_{q^{\prime} n^{\prime} k^{\prime}}^{\left(j^{\prime}\right)}(\mathbf{r})=\delta_{j j^{\prime}} \delta_{n n^{\prime}} \delta\left(q-q^{\prime}\right) \delta\left(k-k^{\prime}\right), \quad j, j^{\prime}=0,1,2
$$

and completeness

$$
\sum_{\operatorname{sgn}\left(E_{n k}\right)} \int_{-\infty}^{\infty} \mathrm{d} q \int_{-\infty}^{\infty} \mathrm{d} k\left[\psi_{q 0 k}^{(0)}(\mathbf{r}) \psi_{q 0 k}^{(0) \dagger}\left(\mathbf{r}^{\prime}\right)+\sum_{n=1}^{\infty} \sum_{j=1,2} \psi_{q n k}^{(j)}(\mathbf{r}) \psi_{q n k}^{(j) \dagger}\left(\mathbf{r}^{\prime}\right)\right]=I \delta\left(\mathbf{r}-\mathbf{r}^{\prime}\right)
$$

With the use of relation

$$
\int_{-\infty}^{\infty} \mathrm{d} q\left[Y_{n}^{q}(y)\right]^{2}=|e B|
$$

expression (48) is readily obtained.

The solution corresponding to a plane wave propagating along the $z$-axis in the opposite direction is written in the case of $e B>0$ in the following form: 


$$
\begin{aligned}
\left.\psi_{q n-k}(\mathbf{r})\right|_{E_{n k}=\omega_{n k}} & =\frac{\mathrm{e}^{\mathrm{i} q x} \mathrm{e}^{-\mathrm{i} k z}}{2 \pi \sqrt{2 \omega_{n k}\left(\omega_{n k}+m\right)}}\left[\tilde{C}_{1}\left(\begin{array}{c}
\left(\omega_{n k}+m\right) Y_{n}^{q}(y) \\
0 \\
-k Y_{n}^{q}(y) \\
\sqrt{2 n e B Y_{n-1}^{q}(y)}
\end{array}\right)\right. \\
& \left.+\tilde{C}_{2}\left(\begin{array}{c}
\left(\omega_{n k}+m\right) Y_{n-1}^{q}(y) \\
\sqrt{2 n e B} Y_{n}^{q}(y) \\
k Y_{n-1}^{q}(y)
\end{array}\right)\right], \quad n \geq 1
\end{aligned}
$$

and

$$
\left.\psi_{q 0-k}^{(0)}(\mathbf{r})\right|_{E_{0 k}=\omega_{0 k}}=\frac{\mathrm{e}^{\mathrm{i} q x} \mathrm{e}^{-\mathrm{i} k z}}{2 \pi \sqrt{2 \omega_{0 k}\left(\omega_{0 k}+m\right)}} \tilde{C}_{0} Y_{0}^{q}(y)\left(\begin{array}{c}
\omega_{0 k}+m \\
0 \\
-k \\
0
\end{array}\right),
$$

or, for the opposite sign of energy,

$$
\begin{aligned}
\left.\psi_{q n-k}(\mathbf{r})\right|_{E_{n k}=-\omega_{n k}} & =\frac{\mathrm{e}^{-\mathrm{i} q x} \mathrm{e}^{\mathrm{i} k z}}{2 \pi \sqrt{2 \omega_{n k}\left(\omega_{n k}+m\right)}}\left[C_{1}\left(\begin{array}{c}
-k Y_{n}^{-q}(y) \\
-\sqrt{2 n e B} Y_{n-1}^{-q}(y) \\
\left(\omega_{n k}+m\right) Y_{n}^{-q}(y) \\
0
\end{array}\right)\right. \\
& \left.+C_{2}\left(\begin{array}{c}
-\sqrt{2 n e B} Y_{n}^{-q}(y) \\
k Y_{n-1}^{-q}(y) \\
0 \\
\left(\omega_{n k}+m\right) Y_{n-1}^{-q}(y)
\end{array}\right)\right], \quad n \geq 1
\end{aligned}
$$

and

$$
\left.\psi_{q 0-k}^{(0)}(\mathbf{r})\right|_{E_{0 k}=-\omega_{0 k}}=\frac{\mathrm{e}^{-\mathrm{i} q x} \mathrm{e}^{\mathrm{i} k z}}{2 \pi \sqrt{2 \omega_{0 k}\left(\omega_{0 k}+m\right)}} C_{0} Y_{0}^{-q}(y)\left(\begin{array}{c}
-k \\
0 \\
\omega_{0 k}+m \\
0
\end{array}\right) .
$$

\section{Appendix B. Abel-Plana summation formula}

Condition (80) is rewritten as

$$
P_{+}\left(k_{l}\right)=0, \quad E_{n l}>0,
$$


or

$$
P_{-}\left(k_{l}\right)=0, \quad E_{n l}<0,
$$

where

$$
P_{ \pm}(u)=\cos (u a)+\frac{ \pm \omega_{n u} \cos \tilde{\varphi}-m \cos \varphi}{u \sin \tilde{\varphi}} \sin (u a)
$$

and, see (47),

$$
\omega_{n u}=\sqrt{u^{2}+2 n|e B|+m^{2}} .
$$

We assign labels $l=0,1,2, \ldots$, to the consecutively increasing positive roots of (B.1) or (B.2), $k_{l}>0$; appropriately, labels $l=-1,-2, \ldots$, are assigned to the consecutively decreasing negative roots of (B.1) or (B.2), $k_{l}<0$. Then one can write

$$
\sum_{\operatorname{sgn}\left(E_{n l}\right)} \sum_{l=0}^{\infty} f\left(k_{l}^{2}\right)=\frac{1}{2} \sum_{\operatorname{sgn}\left(E_{n l}\right)} \sum_{l=-\infty}^{\infty} f\left(k_{l}^{2}\right)=\frac{a}{4 \pi} \int_{C_{=}} \mathrm{d} u f\left(u^{2}\right)\left[G_{+}(u)+G_{-}(u)\right],
$$

where

$$
G_{ \pm}(u)=1+\frac{\mathrm{i}}{a} \frac{\mathrm{d}}{\mathrm{d} u} \ln P_{ \pm}(u)
$$

and contour $C_{=}$on the complex $u$-plane consists of two parallel infinite lines going closely on the lower and upper sides of the real axis, see Fig.1. An explicit form of functions $G_{+}(u)$ and $G_{-}(u)$ is

$$
G_{ \pm}(u)=\frac{\left[u+\mathrm{i} h_{ \pm}(u)\right] \mathrm{e}^{-\mathrm{i} u a}+\mathrm{i} \sin (u a) \frac{u}{a} \frac{\mathrm{d}}{\mathrm{d} u}\left[\frac{h_{ \pm}(u)}{u}\right]}{u \cos (u a)+h_{ \pm}(u) \sin (u a)}
$$

where

$$
h_{ \pm}(u)=\frac{ \pm \omega_{n u} \cos \tilde{\varphi}-m \cos \varphi}{\sin \tilde{\varphi}} .
$$

Since the numerators of $G_{+}(u)$ and $G_{-}(u)$ contribute to the integral in (B.5) at values $u=k_{l}$ only, one may change the numerators with the use of relations (B.1) and (B.2), respectively. In this way we define

$$
\tilde{G}_{ \pm}(u)=\frac{\left[u+\mathrm{i} h_{ \pm}(u)\right] \mathrm{e}^{-\mathrm{i} u a}+\mathrm{i} g_{ \pm}(u)}{u \cos (u a)+h_{ \pm}(u) \sin (u a)}
$$

where

$$
g_{ \pm}(u)=\frac{\left( \pm \frac{\omega_{n 0}^{2}}{\omega_{n u}} \cos \tilde{\varphi}-m \cos \varphi\right) h_{ \pm}(u)}{a \cos (u a) \sin \tilde{\varphi}\left[u^{2}+h_{ \pm}^{2}(u)\right]}
$$




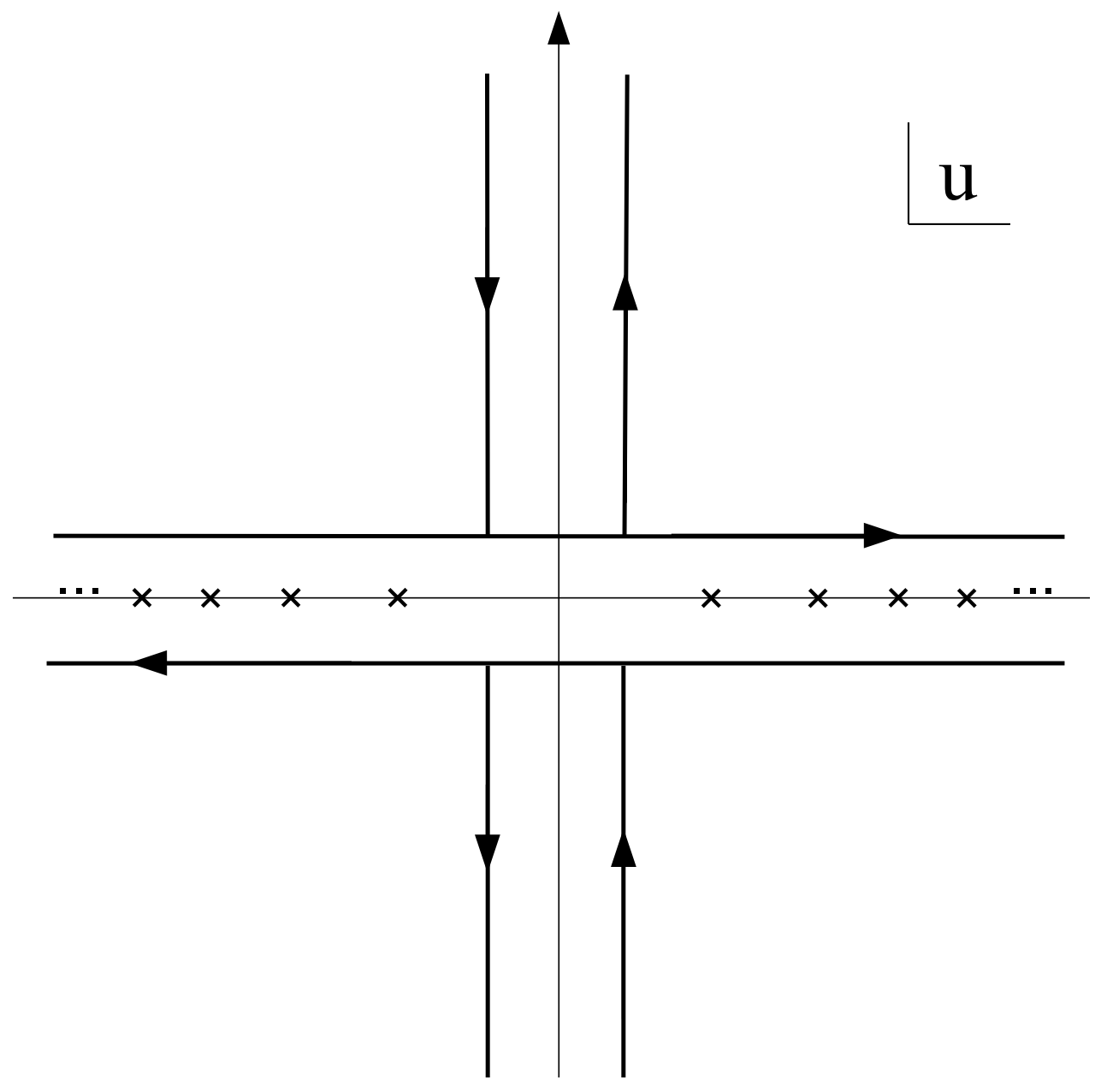

Figure 1: Contours $C_{-}, C_{\sqcap}$ and $C_{\sqcup}$ on the complex $u$-plane; the positions of poles of function $G_{+}(\bar{u})+G_{-}(u)$ are indicated by crosses. 
However, additional simple poles at $u=0$ and at $\cos (u a)=0$ are in $\tilde{G}_{ \pm}(u)$ (B.9), as compared to $G_{ \pm}(u)$ (B.7). Subtracting the contribution of these poles, we obtain identity

$$
\int_{C_{=}} \mathrm{d} u f\left(u^{2}\right)\left[G_{+}(u)+G_{-}(u)\right]=\int_{C_{=}} \mathrm{d} u f\left(u^{2}\right)\left[\tilde{G}_{+}(u)+\tilde{G}_{-}(u)-\frac{2 \mathrm{i}}{u a}+D_{+}(u)+D_{-}(u)\right],
$$

where

$$
\begin{gathered}
D_{ \pm}(u)=\frac{\mathrm{e}^{-\mathrm{i} u a}}{a \cos (u a)} d_{ \pm}(u), \\
d_{ \pm}(u)=\frac{ \pm \frac{\omega_{n 0}^{2}}{\omega_{n u}} \cos \tilde{\varphi}-m \cos \varphi}{\sin \tilde{\varphi}\left[u^{2}+h_{ \pm}^{2}(u)\right]} .
\end{gathered}
$$

Separating explicitly the contribution of the subtracted pole at $u=0$, we consider the remaining integral on the right-hand side of (B.11) by deforming the parts of contour $C_{=}$into the lower and upper parts of the $u$-plane. Note that function $\tilde{G}_{+}(u)+\bar{D}_{+}(u)+\tilde{G}_{-}(u)+D_{-}(u)$, in addition to poles at $u=k_{l}$, has also poles at $u= \pm \mathrm{i}\left(m \cos \varphi \sin \tilde{\varphi}+\cos \tilde{\varphi} \sqrt{2 n|e B|+m^{2} \sin ^{2} \varphi}\right)$ and $u=$ $\pm \mathrm{i}\left(m \cos \varphi \sin \tilde{\varphi}-\cos \tilde{\varphi} \sqrt{2 n|e B|+m^{2} \sin ^{2} \varphi}\right)$. Assuming that all singularities of $f\left(u^{2}\right)$ as a function of complex variable $u$ lie on the imaginary axis at some distances from the origin, we get

$$
\begin{gathered}
\int_{C_{=}} \mathrm{d} u f\left(u^{2}\right)\left[G_{+}(u)+G_{-}(u)\right]=\int_{C_{\Pi}} \mathrm{d} u f\left(u^{2}\right)\left[\tilde{G}_{+}(u)+D_{+}(u)+\tilde{G}_{-}(u)+D_{-}(u)\right] \\
\quad+\int_{C_{\sqcup}} \mathrm{d} u f\left(u^{2}\right)\left[\tilde{G}_{+}(u)+D_{+}(u)+\tilde{G}_{-}(u)+D_{-}(u)\right]-\frac{4 \pi}{a} f(0), \quad \text { (B.14) }
\end{gathered}
$$

where contours $C_{\sqcap}$ and $C_{\sqcup}$ enclose the lower and upper imaginary semiaxes, see Fig.1. In view of obvious relation

$$
\lim _{k \rightarrow 0+}(k \pm \mathrm{i} \kappa)^{2}=\lim _{k \rightarrow 0+}(-k \mp \mathrm{i} \kappa)^{2}=( \pm \mathrm{i} \kappa)^{2}
$$

for real positive $k$ and $\kappa$, we obtain 


$$
\begin{aligned}
\int_{C_{=}} \mathrm{d} u & f\left(u^{2}\right)\left[G_{+}(u)+G_{-}(u)\right]=\mathrm{i} \int_{0}^{\infty} \mathrm{d} \kappa\left\{f\left[(-\mathrm{i} \kappa)^{2}\right]-f\left[(\mathrm{i} \kappa)^{2}\right]\right\} \\
& \times\left[\tilde{G}_{+}(-\mathrm{i} \kappa)+D_{+}(-\mathrm{i} \kappa)+\tilde{G}_{-}(-\mathrm{i} \kappa)+D_{-}(-\mathrm{i} \kappa)\right. \\
- & \left.\tilde{G}_{+}(\mathrm{i} \kappa)-D_{+}(\mathrm{i} \kappa)-\tilde{G}_{-}(\mathrm{i} \kappa)-D_{-}(\mathrm{i} \kappa)\right]-\frac{4 \pi}{a} f(0) .
\end{aligned}
$$

Taking account for relations

$$
\tilde{G}_{+}(-\mathrm{i} \kappa)+\tilde{G}_{-}(-\mathrm{i} \kappa)+\tilde{G}_{+}(\mathrm{i} \kappa)+\tilde{G}_{-}(\mathrm{i} \kappa)=4
$$

and

$$
\begin{gathered}
D_{+}(-\mathrm{i} \kappa)+D_{-}(-\mathrm{i} \kappa)+D_{+}(\mathrm{i} \kappa)+D_{-}(\mathrm{i} \kappa) \\
=\frac{2}{a}\left[d_{+}(-\mathrm{i} \kappa)+d_{-}(-\mathrm{i} \kappa)\right] \equiv \frac{2}{a}\left[d_{+}(\mathrm{i} \kappa)+d_{-}(\mathrm{i} \kappa)\right],
\end{gathered}
$$

we further obtain

$$
\begin{gathered}
\int_{C_{=}} \mathrm{d} u f\left(u^{2}\right)\left[G_{+}(u)+G_{-}(u)\right]=2 \mathrm{i} \int_{0}^{\infty} \mathrm{d} \kappa\left\{f\left[(-\mathrm{i} \kappa)^{2}\right]-f\left[(\mathrm{i} \kappa)^{2}\right]\right\} \\
\quad \times\left[\tilde{G}_{+}(-\mathrm{i} \kappa)+D_{+}(-\mathrm{i} \kappa)+\tilde{G}_{-}(-\mathrm{i} \kappa)+D_{-}(-\mathrm{i} \kappa)\right] \\
\left.-2 \mathrm{i} \int_{0}^{\infty} \mathrm{d} \kappa f\left[(-\mathrm{i} \kappa)^{2}\right)\right]\left\{2+\frac{1}{a}\left[d_{+}(-\mathrm{i} \kappa)+d_{-}(-\mathrm{i} \kappa)\right]\right\} \\
\left.+2 \mathrm{i} \int_{0}^{\infty} \mathrm{d} \kappa f\left[(\mathrm{i} \kappa)^{2}\right)\right]\left\{2+\frac{1}{a}\left[d_{+}(\mathrm{i} \kappa)+d_{-}(\mathrm{i} \kappa)\right]\right\}-\frac{4 \pi}{a} f(0) .
\end{gathered}
$$

By rotating the paths of integration in the last and before the last integrals in (B.18) by $90^{\circ}$ in the clockwise and anticlockwise directions, respectively, we finally get 


$$
\begin{aligned}
\int_{C_{=}} \mathrm{d} u & f\left(u^{2}\right)\left[G_{+}(u)+G_{-}(u)\right]=2 \mathrm{i} \int_{0}^{\infty} \mathrm{d} \kappa\left\{f\left[(-\mathrm{i} \kappa)^{2}\right]-f\left[(\mathrm{i} \kappa)^{2}\right]\right\} \\
& \times\left[\tilde{G}_{+}(-\mathrm{i} \kappa)+D_{+}(-\mathrm{i} \kappa)+\tilde{G}_{-}(-\mathrm{i} \kappa)+D_{-}(-\mathrm{i} \kappa)\right] \\
+ & 4 \int_{0}^{\infty} \mathrm{d} k f\left(k^{2}\right)\left\{2+\frac{1}{a}\left[d_{+}(k)+d_{-}(k)\right]\right\}-\frac{4 \pi}{a} f(0) .
\end{aligned}
$$

The sense of going over from function $G_{ \pm}(u)$ to functions $\tilde{G}_{ \pm}(u)$ and $D_{ \pm}(u)$ is that $\tilde{G}_{ \pm}(-\mathrm{i} \kappa)+D_{ \pm}(-\mathrm{i} \kappa)$, unlike $G_{ \pm}(-\mathrm{i} \kappa)$, is exponentially decreasing at large values of $\kappa$. Defining

$$
\Lambda(\kappa)=\frac{1}{4}\left[\tilde{G}_{+}(-\mathrm{i} \kappa)+D_{+}(-\mathrm{i} \kappa)+\tilde{G}_{-}(-\mathrm{i} \kappa)+D_{-}(-\mathrm{i} \kappa)\right]
$$

which is explicitly given by (88), substituting the explicit form for $d_{+}(k)+$ $d_{-}(k)$ and recalling (B.5), we rewrite (B.19) into the form given by (87). It should be noted that the contribution of poles on the imaginary axis at $u= \pm \mathrm{i}\left(m \cos \varphi \sin \tilde{\varphi}+\cos \tilde{\varphi} \sqrt{2 n|e B|+m^{2} \sin ^{2} \varphi}\right)$ and $u= \pm \mathrm{i}(m \cos \varphi \sin \tilde{\varphi}-$ $\left.\cos \tilde{\varphi} \sqrt{2 n|e B|+m^{2} \sin ^{2} \varphi}\right)$ is cancelled.

\section{References}

[1] A.Chodos, R.L.Jaffe, K.Johnson, C.B.Thorn and V.Weisskopf, Phys. Rev. D 9, 3471 (1974).

[2] A.Chodos, R.L.Jaffe, K.Johnson and C.B.Thorn, Phys. Rev. D 10, 2599 (1974).

[3] T.De Grand, R.L.Jaffe, K.Johnson and J.Kiskis, Phys. Rev. D 12, 2060 (1975).

[4] K.Johnson, Acta Phys. Pol. B 6, 865 (1975).

[5] W.Heisenberg, Z. Phys. 90, 209 (1934).

[6] H.Euler and B.Kockel, Naturwissensch. 23, 246 (1935).

[7] W.Heisenberg and H.Euler, Z. Phys. 98, 714 (1936). 
[8] V.S.Weisskopf, Kong. Dans. Vid. Selsk. Mat-Fys. Medd. 14 No.6 (1936).

[9] J.Schwinger, Phys. Rev. 82, 662 (1951).

[10] G.V.Dunne, 'Heisenberg-Euler effective lagrangians: Basics and extensions'. In: Ian Kogan Memorial Collection 'From Fields to Strings: Circumnavigating Theoretical Physics'. Ed. by M.Shifman, A.Vainshtein and J.Wheater (World Scientific, Singapore, 2004) vol.1, pp. 445-522.

[11] M.H.Al-Hashimi and U.-J.Wiese, Annals Phys. 327, 1 (2012).

[12] Yu.A.Sitenko, Phys. Rev. D 91, 085012 (2015).

[13] J.von Neumann, Mathematische Grundlagen der Quantummechanik (Springer, Berlin, 1932).

[14] N.I.Akhiezer and I.M.Glazman, Theory of Linear Operators in Hilbert Space (Pitman, Boston, 1981).

[15] H.B.G.Casimir, Proc. Kon. Ned. Akad. Wetenschap B 51, 793 (1948),

[16] K.A.Milton, The Casimir Effect: Physical Manifestations of Zero-Point Energy (World Scientific, River Edge, 2001).

[17] M.Bordag, G.L.Klimchitskaya, U.Mohideen and V.M.Mostepanenko, Advances in the Casimir Effect (Oxford University Press, Oxford, 2009).

[18] S.Mereghetti, Astron. Astrophys. Rev. 15, 225 (2008).

[19] D.Grasso and H.R.Rubinstein, Phys. Rep. 348, 163 (2001).

[20] A.I.Akhiezer and V.B.Berestetskij, Quantum Electrodynamics (Interscience, New York, 1965). 